Pacific Journal of Mathematics

A TWO SIDED APPROXIMATION THEOREM FOR 2-SPHERES 


\title{
A TWO SIDED APPROXIMATION THEOREM FOR 2-SPHERES
}

\author{
W. T. EATON
}

The side approximation theorem proved by $R$. H. Bing and later improved by F. M. Lister states that a sphere $S$ topologically embedded in Euclidean three space can be $\varepsilon$ approximated with polyhedral spheres $g(S)$ and $h(S)$ such that $g\left(S-\cup G_{i}\right) \subset \operatorname{Int} S, g\left(G_{i}\right) \cap S \subset G_{i}, h\left(S-\cup H_{i}\right) \subset$ Ext $S$, and $h\left(H_{i}\right) \cap S \subset H_{i}$ where $\left\{G_{i}\right\}$ and $\left\{H_{i}\right\}$ are respectively finite collections of disjoint $\varepsilon$-disks in $S$. In this article the theorem is strengthened by showing that the sets $\cup G_{i}$ and $\cup H_{i}$ may also be taken to be disjoint.

The theorem is a generalization of R. H. Bing's side approximation theorem [2], [3] and is used [7], [8] to distinguish certain decomposition spaces from 3-manifolds.

The proof is divided into two main parts. In §2, Theorem 2.1 is reduced to Corollary 6.1 of $\S 6$ using techniques that are consequences of Bing's side approximation theorem. The proof of Corollary 6.1 is the main topic of this paper. The proof depends on Lemma 5.15 and Theorem 5.16 of $\S 5$ which give the combinatorial structure of collections of components associated with the general position of two 2-spheres in a 3-manifold. The proofs of Lemma 5.15 and Theorem 5.16 depend in turn on the concepts of separation complexes and winding functions introduced, respectively, in $\S \S 3$ and 4 .

2. Reduction to geometry. The main result of this paper is Theorem 2.1 stated below. The purpose of this section is to reduce the proof of Theorem 2.1 to Corollary 6.1 of $\S 6$.

THEOREM 2.1. If $S$ is a 2-sphere in $E^{3}$ and $\varepsilon$ is a positive number then there exists a finite collection $\left\{G_{1}, \cdots, G_{n}, H_{1}, \cdots, H_{n}\right\}$ of disjoint $\varepsilon$-disks in $S$ and $\varepsilon$-homeomorphisms $g, h: S \rightarrow E^{3}$ such that

(2.1.1) $g\left(S-\cup \operatorname{Int} G_{i}\right) \subset \operatorname{Int} S$,

(2.1.2) $g\left(G_{i}\right) \cap S \subset \operatorname{Int} G_{i}$,

(2.1.3) $\quad h\left(S-\cup \operatorname{Int} H_{i}\right) \subset \operatorname{Ext} S$, and

(2.1.4) $h\left(H_{i}\right) \cap S \subset \operatorname{Int} H_{i}$.

Furthermore, $g(S)$ and $h(S)$ may be chosen to be polyhedral and disjoint.

Proof. It follows from F. M. Lister's approximation theorem [9] that there exists a disjoint collection of $(\varepsilon / 3)$-disks $C_{1}, \cdots, C_{n}$ in $S$ and $(\varepsilon / 3)$-homeomorphisms $g_{0}, h_{0}: S \rightarrow E^{3}$ such that $g_{0}\left(S-\cup \operatorname{Int} C_{i}\right) \subset$ 
Int $S, h_{0}\left(S-\cup \operatorname{Int} C_{i}\right) \subset \operatorname{Ext} S, g_{0}\left(C_{i}\right) \cap S \subset \operatorname{Int} C_{i}, h_{0}\left(C_{i}\right) \cap S \subset \operatorname{Int} C_{i}$, and $g_{0}(S), h_{0}(S)$ are polyhedral. Let $V_{1}, \cdots, V_{n}$ be a collection of disjoint open $\varepsilon$-subsets of $E^{3}$ such that $C_{i} \cup g_{0}\left(C_{i}\right) \cup h_{0}\left(C_{i}\right) \subset V_{i}$ There is no loss in generality [4] in assuming that $\mathrm{Bd} C_{i}$ is tame.

The required disks $G_{i}, H_{i}$ will be disjoint subdisks of $C_{i}$ and the required homeomorphisms $g, h$ will have the properties that

$$
g\left|S-\cup C_{i}=g_{0}\right| S-\cup C_{i}, g\left(C_{i}\right) \subset V_{i}, h\left|S-\cup C_{i}=h_{0}\right| S-\cup C_{i}
$$

and $h\left(C_{i}\right) \subset V_{i}$. All of the adjusting is done in the $V_{i}$ 's one-at-a-time and independently. Let $i$ be fixed and to simplify notation let $C=$ $C_{i}$, and $V=V_{i}$. Let $E_{0}=h_{0}(C)$ and $E_{1}=g_{0}(C)$. By standard scissorand-paste techniques we may assume that $E_{0} \cap E_{1}=\varnothing$.

By the polyhedral approximation theorem [1] we may polyhedrally approximate $C$ to obtain a disk $E^{\prime}$ such that $E^{\prime} \cap(S-\operatorname{Int} C)=\mathrm{Bd} E^{\prime}=$ $\mathrm{Bd} C, E^{\prime} \subset V, E^{\prime} \cap h_{0}(S) \subset \operatorname{Int} E_{0}$, and $E^{\prime \prime} \cap g_{0}(S) \subset \operatorname{Int} E_{1}$. We may assume that $E^{\prime \prime}$ and $E_{0} \cup E_{1}$ are in general position. Let $E^{\prime \prime}$ be the component of $E^{\prime}-\left(E_{0} \cup E_{1}\right)$ that contains $\mathrm{Bd} E^{\prime}$. By filling in the holes of $E^{\prime \prime}$ with disks in $E_{0} \cup E_{1}$ pushed slightly to the sides of $E_{0} \cup E_{1}$, we obtain a polyhedral disk $E$ such that $E \cap(S-\operatorname{Int} C)=$ $\mathrm{Bd} E=\mathrm{B} d C, E \subset V$, and $E \cap h_{0}(S)=E \cap g_{0}(S)=\varnothing$.

Let $\delta=(1 / 9) \rho\left(E, E_{0} \cup E_{1}\right)$. By [5, Theorem 9.1] there exists a tame Sierpinski curve $X \subset C$ such that $\mathrm{Bd} C \subset X$ and the components $X_{1}, X_{2}, \cdots$ of $C-X$ each have diameter less than $\delta$. By the polyhedral approximation theorem [1], there exist disks $Y_{i}$ such that $\operatorname{Bd} Y_{i}=\operatorname{Bd} X_{i}, D=X \cup\left(\cup Y_{i}\right)$ is a disk, Diam $Y_{i}<\delta, Y_{i} \subset V, X_{j} \cap Y_{i}=$ $\varnothing$ if $i \neq j$, and $Y_{i}$ is locally polyhedral modulo $\mathrm{Bd} Y_{i}$. It follows from [5] that $D$ is tame. Keeping points of $E^{3}-V, g_{0}(S)-\operatorname{Int} E_{1}$, $S$ - Int $E$, and $h_{0}(S)$ - Int $E_{0}$ fixed and moving no point as far as $\delta$, we first move $E_{0} \cup E \cup E_{1}$ into general position with respect to $D$ with $\mathrm{Bd} E=\mathrm{Bd} D$ and then with a move "parallel" to $D$, we push $\left(\left(E_{0} \cup E \cup E_{1}\right) \cap D\right)-\mathrm{Bd} E$ to the inaccessible part of $X$ (see [6] for more details).

The preceding adjustments enable us assume without loss of generality that $E_{0}, E, E_{1}$ are polyhedral and disjoint, $E_{0} \cup E \cup E_{1}$ is in general position with respect to $D$ with $\left(\left(E_{0} \cup E \cup E_{1}\right) \cap D\right)-\mathrm{Bd} E$ in the inaccessible part of $X$ and

no $\mathrm{Cl} X_{i}$ intersects more than one of $E_{0}, E, E_{1}$.

In order to apply Corollary 6.1 we add the ideal point $\infty$ to $E^{3}$, let $T$ be the 2-sphere $(S-C) \cup D, U_{i}$ be the component of $S^{3}-T$ containing $\mathrm{Bd} E_{i}$, and let $E, E_{0}, E_{1}$ of Corollary 6.1 be as above. Let $D_{0}, D_{1}$ be the singular disks of the conclusion of Corollary 6.1.

Since $\left(E_{0} \cup E_{1}\right) \cap D$ lies in the inaccessible part of $X$ there is a 
natural open disk-with-holes $B^{*}$ in $S$ associated with each $B \in \mathscr{D}_{i}$; namely, let $B^{*}$ be obtained from $B$ by replacing each $Y_{j} \subset B$ with Cl $X_{j}$. If $\mathscr{D}_{i}^{*}=\left\{B^{*} \mid B \in \mathscr{D}_{i}\right\}$ then $D_{i}^{*}=\cup\left\{\mathrm{Cl} B \mid B \in \mathscr{D}_{i}^{*} \cup \mathscr{C}_{i}\right\}$ are clearly singular disks, and $D_{0}^{*} \cap D_{1}^{*}=\varnothing$ since $D_{0} \cap D_{1}=\varnothing$ and each $X_{j}$ intersects at most one of $E_{0}$ and $E_{1}$. Some of the $X_{j}$ 's may pass through elements of $\mathscr{C}_{i}$; thus, $D_{i}^{*}$ may not lie in the closure of a complementary domain of $S$. In the next paragraph we adjust the $D_{i}^{*}$ 's so that each does lie in the closure of a complementary domain of $S$ while retaining the property $D_{0}^{*} \cap D_{1}^{*}=\varnothing$. We first observe that no element of $\mathscr{D}_{1-i}^{*}$ intersects an element of $\mathscr{E}_{i}$. For suppose that $X_{j} \subset B^{*} \in \mathscr{D}_{1-i}^{*}, A \in \mathscr{E}_{i}$ and $X_{j} \cap A \neq \varnothing$ then, since $X_{j}$ is connected and $X_{j} \cap Y_{k}=\varnothing$ if $k \neq j$, there exists a component $K$ of $X_{j} \cap U_{i}$ such that $K \cap A \neq \varnothing$ and $Y_{j} \cap \mathrm{Cl} K \neq \varnothing$. Since $Y_{j} \subset B$ and $E$ separates $A$ from $B$ in $\mathrm{Cl} U_{i}$ by (6.1.2), $\mathrm{Cl} K$ intersects $E$. Thus, $\mathrm{Cl} X_{j}$ intersects both $E$ and $E_{i}$ and this contradicts (2.2).

For $i=0,1$, let $f_{1}^{*}$ be a map on $E_{i}$ with the property that $f_{1}^{*}\left(E_{i}\right)=D_{1}^{*}$ and $f_{i}^{*} \mid \operatorname{Bd} E_{i}=1$. Let $E_{0}^{*}$ be the component of

$$
E_{0} \cap\left(f_{0}^{*}\right)^{-1}\left(\mathrm{Cl}(\operatorname{Ext} S) \cap D_{0}^{*}\right)
$$

that contains $\operatorname{Bd} E_{0}$ and let $E_{1}^{*}$ be the component of

$$
E_{1} \cap\left(f_{0}^{*}\right)^{-1}\left(\mathrm{Cl}(\operatorname{Int} S) \cap D_{1}^{*}\right)
$$

that contains $\mathrm{Bd} E_{1}$. By the observations of the previous paragraph there exist subsequences $\left\{X_{k}^{0}\right\},\left\{X_{j}^{1}\right\}$, of $\left\{X_{i}\right\}$ such that $\mathrm{Cl}\left(\cup X_{k}^{0}\right) \cap$ $\mathrm{Cl}\left(\cup X_{j}^{1}\right)=\varnothing$ and $f_{0}^{*}\left(\left(\mathrm{Bd} E_{0}^{*}\right)-\operatorname{Bd} E_{0}\right) \subset \mathrm{Cl}\left(\cup X_{k}^{0}\right), f_{1}^{*}\left(\left(\mathrm{Bd} E_{1}^{*}-\mathrm{Bd} E_{0}\right) \subset\right.$ $\mathrm{Cl}\left(\cup X_{j}^{1}\right)$. Also, if $C_{0}$ is a component of $E_{0}-E_{0}^{*}$ then there exists a unique $X_{k}^{0}$ such that $f_{0}^{*}\left(\mathrm{Bd} C_{0}\right) \subset X_{k}^{0}$. Let $E_{0}^{k}=\operatorname{Cl}\left(\cup\left\{C_{0} \mid f_{0}^{*}\left(\mathrm{Bd} C_{0}\right) \subset X_{k}^{0}\right\}\right)$. We apply the Tietze-Extension-Theorem to normal space $E_{0}^{k}$, closed set $\mathrm{Bd} E_{0}^{k}$, map $f_{0}^{*} \mid \mathrm{Bd} E_{0}^{k}$, and disk $\mathrm{Cl} X_{k}^{0}$ to extend $f_{0}^{*} \mid E_{0}^{*}$ to $E_{0}^{*} \cup E_{0}^{k}$. Since $\left\{\mathrm{Cl} X_{k}^{o}\right\}$ is a null-sequence, extending $f_{0}^{*} \mid E_{0}^{*}$ to each $E_{0}^{k}$ in the above manner and piecing together the resulting maps yeilds a map $f_{0}^{\prime}: E_{0} \rightarrow \mathrm{Cl}\left(\right.$ Ext S). In the same way, we construct a map $f_{1}^{\prime}: E_{1} \rightarrow$ $\mathrm{Cl}($ Int $S)$. The maps $f_{0}^{\prime}: E_{0} \rightarrow \mathrm{Cl}(\operatorname{Ext} S), f_{1}^{\prime}: E_{1} \rightarrow \mathrm{Cl}(\operatorname{Int} S)$ have the following properties $f_{i}^{\prime} \mid \mathrm{Bd} E_{i}=1, f_{0}^{\prime}\left(E_{0}-\mathrm{Bd} E_{0}\right) \cap h_{0}(S-\operatorname{Int} C)=\varnothing$, $f_{1}^{\prime}\left(E_{1}-\operatorname{Bd} E_{1}\right) \cap g_{0}(S-\operatorname{Int} C)=\varnothing, f_{i}^{\prime}\left(E_{i}\right) \subset V$, and most importantly $f_{0}^{\prime}\left(E_{0}\right) \cap f_{1}^{\prime}\left(E_{1}\right)=\varnothing$.

By the results of [5], for each positive number $\delta$ there exist $\delta$ maps $\alpha: \mathrm{Cl}(\operatorname{Int} S) \rightarrow \mathrm{Cl}(\operatorname{Int} S), \beta: \mathrm{Cl}(\operatorname{Ext} \delta) \rightarrow \mathrm{Cl}(\operatorname{Ext} S)$ such that $S \cap$ $\alpha(\mathrm{Cl}(\operatorname{Int} S)), S \cap \beta(\mathrm{Cl}(\operatorname{Int} S))$ are 0 -dimensional and $\alpha \mid(\operatorname{Int} S)-N=$ $1, \beta \mid($ Ext $S)-N=1$ where $N$ is an arbitrary neighborhood of $S$. By choosing $\delta$ and $N$ appropriately, in particular $\delta$ less than $(1 / 2) \rho\left(f_{0}^{\prime}\left(E_{0}\right)\right.$, $\left.f_{1}^{\prime}\left(E_{1}\right)\right)$, we select maps $\alpha, \beta$ so that $\alpha f_{1}^{\prime}\left(E_{1}\right) \subset \mathrm{Cl}(\operatorname{Int} S), \alpha f_{1}^{\prime} \mid \mathrm{Bd} E_{1}=$ 1, $\alpha f_{1}^{\prime}\left(E_{1}-\operatorname{Bd} E_{1}\right) \cap g_{0}(S-\operatorname{Int} C)=\varnothing, \alpha f_{1}^{\prime}\left(E_{1}\right) \subset V, \beta f_{0}^{\prime}\left(E_{0}\right) \subset \operatorname{Cl}(\operatorname{Ext} S)$, 
$\beta f_{0}^{\prime} \mid \operatorname{Bd} E_{0}=1, \beta f_{0}^{\prime}\left(E_{0}-\operatorname{Bd} E_{0}\right) \cap h_{0}(S-\operatorname{Int} C)=\varnothing, \beta f_{0}^{\prime}\left(E_{0}\right) \subset V$, and $\alpha f_{0}^{\prime}\left(E_{1}\right) \cap S, \beta f_{0}^{\prime}\left(E_{0}\right) \cap S$ are disjoint compact 0-dimensional subsets of Int $C$.

Let $G, H$ be disjoint disks in Int $C$ such that $\alpha f_{1}^{\prime}\left(E_{1}\right) \cap S \subset \operatorname{Int} G$ and $\beta f_{0}^{\prime}\left(E_{0}\right) \cap S \subset$ Int $H$. We apply Dehn's lemma [10] to each of $\alpha f_{1}^{\prime}\left(E_{1}\right)$ and $\beta f_{0}^{\prime}\left(E_{0}\right)$ in a small neighborhood and each to obtain real disks $E_{1}^{\prime}$ and $E_{0}^{\prime}$, respectively. We obtain the maps $g, h$ of the conclusion by adjusting $g_{0}, h_{0}$ in $V$ so that $g_{0}(G)=E_{1}^{\prime}$ and $h_{0}(H)=E_{0}^{\prime}$.

3. Separation complexes. In $\S 2$ we reduced Theorem 2.1 to Corollary 6.1 of Theorem 5.16 in $\S \S 6$ and 5, respectively. In this section we develop the concept of a separation complex which is used extensively in $\S \S 4,5$, and 6 . We begin by reminding the reader of the definition of a collar and then defining a separation complex.

Definition 3.1. If $M$ is a $P L$ manifold and $N$ is a $P L$ manifold in $\mathrm{Bd} M$ then a collar of $N$ in $M$ is a $P L$ embedding $h$ of $N \times I$ into $M$ such that $h(y \times 0)=y$, and $h(N \times(0,1]) \subset \operatorname{Int} M$.

Definition 3.2. If $N$ is a compact, connected, orientable 2-manifold in $S^{3}$ with nonempty boundary and $U$ is a side of $N$ (i.e., $U$ is the interior of a compact, connected 3-manifold $M$ in $S^{3}$ such that $N \subset \mathrm{Bd} M$ ) then a collar of $N$ to the $U$ side of $N$ is a $P L$ embedding $h$ of $N \times I$ into $\mathrm{Cl} U$ such that $h(y \times 0)=y$, and $h(N \times$ $(0,1]) \subset U$.

DEFINITION 3.3. A separation complex is a finite collection $K=$ $K_{1} \cup K_{2} \cup K_{3}$ of sets in $S^{3}$ such that

(3.3.1) $K_{1}$ is a collection of disjoint $P L$ simple closed curves, called separation 1-simplices,

(3.3.2) $K_{2}$ is a collection of compact, connected, orientable, $P L$ 2-manifolds with nonempty boundary, call separation 2-simplices, such that

(3.3.2.1) if $\sigma \in K_{2}$ and $\dot{\sigma}$ is the collection of separation 1-simplices of $K$ intersecting $\sigma$, then $\mathrm{Bd} \sigma=\cup\{\tau \mid \tau \in \dot{\sigma}\}$ and each element of $\dot{\sigma}$ is called a 1 -face of $\sigma$,

(3.3.2.2) if $\sigma_{1}, \sigma_{2} \in K_{2}$ then $\sigma_{1} \cap \sigma_{2}$ is a union of faces of each,

(3.3.3) $K_{3}$ is a collection of compact, connected, $P L$ 3-manifolds with nonempty boundary, called separation 3-simplices, such that

(3.3.3.1) if $\sigma \in K_{3}$ and $\dot{\sigma}$ is the collection of separation 2-simplices of $K$ intersecting $\sigma$, then $\mathrm{Bd} \sigma=\bigcup\{\tau \mid \tau \in \dot{\sigma}\}$ and each element $\tau$ of $\dot{\sigma}$ is called a 2-face of $\sigma$; each separation 1-simplex which intersects $\sigma$ lies in $\mathrm{Bd} \sigma$ 
and is called a 1-face of $\sigma$,

(3.3.3.2) if $\sigma_{1}, \sigma_{2} \in K_{3}$ then $\sigma_{1} \cap \sigma_{2}$ is a union of 1 and 2 faces of each.

DEFINITION 3.4. A subcomplex of a separation complex $K$ is a subcollection $L \subset K$ that is also a separation complex.

LEMma 3.5. If $K$ is a separation complex then $L \subset K$ is a subcomplex of $K$ if and only if $\sigma \in L$ implies $\dot{\sigma} \subset L$.

Notation. If $K$ is a separation complex then $|K|=\bigcup_{\sigma \in K} \sigma$, and if $\sigma \in K$ then $\bar{\sigma}$ denotes the subcomplex of $K$ consisting of $\sigma$ and its faces.

DeFinition 3.6. If $K=K_{1} \cup K_{2} \cup K_{3}, L=L_{1} \cup L_{2} \cup L_{3}$ are separation complexes, then a function $f:|K| \rightarrow|L|$ is a separation isomorphism from $K$ to $L$ if $f$ is one-to-one onto, $\sigma \in K_{i}$ implies $f(\sigma) \in L_{i}$, and $\sigma \in K$ implies $f(\mathrm{Bd} \sigma)=\operatorname{Bd} f(\sigma)$. Note that the function $f \mid \sigma \in K$ need not be a homeomorphism or even continuous. In general $\sigma$ and $f(\sigma)$ are necessarily homeomorphic only if $\sigma \in K_{1}$, in which case they are both simple closed curves.

Definition 3.7. If $K, K^{\prime}$ are separation complexes then $K^{\prime}$ is a subdivision of $K$ if $\left|K^{\prime}\right|=|K|$ and each element of $K$ is a union of elements of $K^{\prime}$.

Suppose $K, L$ are separation complexes, $f$ is a separation isomorphism from $K$ to $L$, and $K^{\prime}$ is a subdivision of $K$. Does there exist a subdivision $L^{\prime}$ of $L$ such that $K^{\prime}$ and $L^{\prime}$ are separation isomorphic? In general the answer is no as Figure 1 indicates. However, for suitably restricted subdivisions $K^{\prime}$ the answer is yes.

DeFinition 3.8. If $K=K_{1} \cup K_{2} \cup K_{3}$ is a separation complex then a subdivision $K^{\prime}=K_{1}^{\prime} \cup K_{2}^{\prime} \cup K_{3}^{\prime}$ of $K$ is a separation subdivision of $K$ if

(3.8.1) if $\sigma \in K_{1}^{\prime}$ then there exists $\tau \in K_{2}$ such that $\sigma \in \dot{\tau}$ or $\sigma \subset$ Int $\tau$ and separates $\tau$,

(3.8.2) if $\sigma \in K_{2}^{\prime}$ then (a) there exists a $\tau \in K_{2}$ such that $\sigma \subset \tau$ or $(b)$ there exists a $\tau \in K_{3}$ such that $\sigma \subset \tau, \sigma \cap \mathrm{Bd} \tau=\mathrm{Bd} \sigma$, and $\sigma$ separates $\tau$.

One easily verifies the equivalence of Definition 3.8 with the following:

DeFinition 3.9. If $K=K_{1} \cup K_{2} \cup K_{3}$ is a separation complex, then a subdivision $K^{\prime}=K_{1}^{\prime} \cup K_{2}^{\prime} \cup K_{3}^{\prime}$ is an elementary separation subdivision of $K$ if there are separation simplices $\sigma \in K_{i}^{\prime}(i=1$ or 2$)$, 


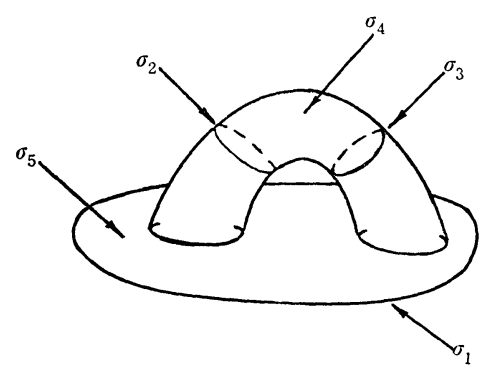

$$
\begin{aligned}
K^{\prime} & =\left\{\sigma_{1}, \sigma_{2}, \sigma_{3}, \sigma_{4}, \sigma_{5}\right\} \\
K & =\left\{\sigma_{1}, \sigma_{4} \cup \sigma_{5}\right\}
\end{aligned}
$$

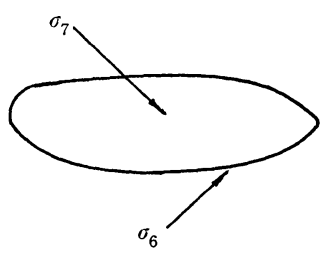

$L=\left\{\sigma_{6}, \sigma_{7}\right\}$

FIGURE 1

$\tau_{1}, \tau_{2} \in K_{i+1}^{\prime}$, and $\tau \in K_{i+1}$ such that

(3.9.1) $\quad K^{\prime}=(K-\{\tau\}) \cup\left\{\tau_{1}, \tau_{2}, \sigma\right\}$

(3.9.2) $\tau=\tau_{1} \cup \tau_{2}$, and

(3.9.3) $\sigma=\tau_{1} \cap \tau_{2}$.

(Note that if $i=2$, then each face of $\sigma$ lies not only in $K_{1}^{\prime}$ but also in $K_{1}$.) A subdivision $K^{\prime \prime}$ of $K$ is a separation subdivision if there is a sequence $K=K_{0}, K_{1}, \cdots, K_{n}=K^{\prime \prime}$ such that, for each $i>0, K_{i}$ is an elementary separation subdivision of $K_{i-1}$.

REMARK. The conditions of Definition 3.8 are often easier to check then those of Definition 3.9. On the other hand, the conditions of Definition 3.9 are easier to use in proofs about separation subdivisions (e.g., see the proof of Lemma 3.10). Separation subdivisions will be obtained in this paper by the methods outlined in Lemmas $3.10,3.13$, and 3.14 .

LeмmA 3.10. Suppose $K=K_{1} \cup K_{2} \cup K_{3}, L=L_{1} \cup L_{2} \cup L_{3}$ are separation complexes, $f$ is a separation isomorphism from $K$ to $L$, and $K^{\prime}=K_{1}^{\prime} \cup K_{2}^{\prime} \cup K_{3}^{\prime}$ is a separation subdivision of $K$. Then there exists a separation subdivision $L^{\prime}=L_{1}^{\prime} \cup L_{2}^{\prime} \cup L_{3}^{\prime}$ of $L$ and a separation isomorphism $f^{\prime}$ of $K^{\prime}$ to $L^{\prime}$ such that $f^{\prime}(\sigma)=f(\sigma)$ if $\sigma \in K$.

Proof. By induction on the number of elementary subdivisions required to change $K$ to $K^{\prime}$, it suffices to prove Lemma 3.10 when $K^{\prime}$ is an elementary separation subdivision of $K$. Let $\sigma, \tau_{1}, \tau_{2}$, and $\tau$ be as (3.9) with (Case A) $\sigma \in K_{1}^{\prime}$ or (Case B) $\sigma \in K_{2}^{\prime}$.

Case A. Let $M$ be a collar of $f\left(\left(\mathrm{Bd} \tau_{1}\right)-\sigma\right)$ in $f(\tau)$ and let $M^{\prime}$ be a 2-manifold-with-boundary in $f(\tau)$ obtained by connecting up the components of $M$ with 2-dimensional tubes in Int $f(\tau)$. The boundary of $M^{\prime}$ is $f\left(\left(\mathrm{Bd} \tau_{1}\right)-\sigma\right) \cup J$ where $J$ is a $P L$ simple closed curve in Int $f(\tau)$. Let $L^{\prime}=L_{1}^{\prime} \cup L_{2}^{\prime} \cup L_{3}^{\prime}$ be defined by $L_{1}^{\prime}=L_{1} \cup\{J\}, L_{2}^{\prime}=$ 
$\left(L_{2}-\{f(\tau)\}\right) \cup\left\{M^{\prime}, \mathrm{Cl}\left(f(\tau)-M^{\prime}\right)\right\}$, and $L_{3}^{\prime}=L_{3}$. Let $f^{\prime}$ be defined by $f^{\prime}|| K \mid-$ Int $\tau=f|| K \mid-$ Int $\tau, f^{\prime} \mid \sigma$ is any one-to-one function onto $J, f^{\prime} \mid$ Int $\tau_{1}$ is any one-to-one function onto Int $M^{\prime}$, and $f^{\prime} \mid$ Int $\tau_{2}$ is any one-to-one function onto $(\operatorname{Int} f(\tau))-M^{\prime}$.

Case B. This case is handled in much the same way as Case A. Let $M$ be a collar of $f\left(\left(\mathrm{Bd} \tau_{1}\right)-\right.$ Int $\left.\sigma\right)$ in $f(\tau)$ and let $M^{\prime}$ be a 3-manifold-with-boundary in $f(\tau)$ obtained by connecting up the components of $M$ with 3-dimensional tubes in Int $f(\tau)$. The boundary of $M^{\prime}$ is $f\left(\left(\operatorname{Bd} \tau_{1}\right)-\sigma\right) \cup J$ where $J$ is a connected $P L$ 2-manifold in $f(\tau)$ such that $J \cap \operatorname{Bd} f(\tau)=\mathrm{Bd} J=f(\mathrm{Bd} \sigma)$. Let $L^{\prime}=L_{1}^{\prime} \cup L_{2}^{\prime} \cup L_{3}^{\prime}$ be defined by $L_{1}^{\prime}=L_{1}, L_{2}^{\prime}=L_{2} \cup\{J\}$, and $L_{3}^{\prime}=\left(L_{3}-\{f(\tau)\}\right) \cup\left\{M^{\prime}, \mathrm{Cl}\left(f(\tau)-M^{\prime}\right)\right\}$. Let $f^{\prime}$ be defined by $f^{\prime}|| K|-\operatorname{Int} \tau=f||K|-\operatorname{Int} \tau, f^{\prime} \mid$ Int $\sigma$ is any one-to-one function onto Int $J, f^{\prime} \mid \operatorname{Int} \tau_{1}$ is any one-to-one function onto Int $M^{\prime}$, and $f^{\prime} \mid \operatorname{Int} \tau_{2}$ is any one-to-one function onto $(\operatorname{Int} f(\tau))-M^{\prime}$.

Notation. If $K$ is a separation complex, $L$ subcomplex of $K$ and $K^{\prime}$ a subdivision of $K$ then $L^{\prime}$ denotes a subdivision of $L$ induced by $K^{\prime}$ given by $L^{\prime}=\left\{\sigma \in K^{\prime}|\sigma \subset| L \mid\right\}$. Note that if $K^{\prime}$ is a separation subdivision of $K$ then $L^{\prime}$ is separation subdivision of $L$.

We are greatly indebted to the referee for the notation $K \bmod W$ in definition 3.12. This concept has simplified our earlier exposition considerably.

DEFINITION 3.11. Let $K=K_{1} \cup K_{2} \cup K_{3}$ denote a separation complex in $S^{3}$ and $W$ a compact $P L$ 2-manifold-with-boundary in $S^{3}$. We shall say that $K$ and $W$ are in general position if the following conditions are satisfied:

(3.11.1) $\quad W \cap\left|K_{1}\right|=\varnothing$.

(3.11.2) If $\tau \in K_{2}$ and $J$ is a component of $W \cap|\tau|$, then either $J$ is a component of $\mathrm{Bd} W$, or $J$ is a simple closed curve in Int $W$ and $\tau$ and $W$ locally cross each other at $J$.

(3.11.3) If $\sigma \in K_{3}$, then $\mathrm{Bd} W \cap \operatorname{Int} \sigma=\varnothing$ and each component of $W \cap|\sigma|$ intersects $\mathrm{Bd} \sigma$.

DeFinition 3.12. If $K$ and $W$ are in general position, then we define $K \bmod W=K_{1}^{\prime} \cup K_{2}^{\prime} \cup K_{3}^{\prime}$ as follows:

(3.12.1) $\quad K_{1}^{\prime}=K_{1} \cup\left\{t \mid\right.$ there exists a $\tau \in K_{2}$ such that $t$ is a component of $W \cap|\tau|\}$.

(3.12.2) $\quad K_{2}^{\prime}=\left\{\mathrm{Cl} Y \mid\right.$ there exists a $\sigma \in K_{3}$ such that $Y$ is a component of $W \cap \operatorname{Int} \sigma\} \cup\left\{\mathrm{Cl} Y \mid\right.$ there exists a $\tau \in K_{2}$ such that $Y$ is a component of $|\tau|-W\}$.

(3.12.3) $\quad K_{3}^{\prime}=\left\{\mathrm{Cl} Z \mid\right.$ there exists a $\sigma \in K_{3}$ such that $Z$ is a component of $|\sigma|-W\}$.

It is not necessarily true that $K \bmod W$ is even a separation com- 
plex (a 2-simplex might intersect the interior of a 3-simplex or a 1-simplex the interior of a 2-simplex). However, we do have the following.

LEMma 3.13. Let $K$ denote a separation complex in $S^{3}$ and $W$ a compact $P L$ 2-manifold-with-boundary in $S^{3}$ such that $K$ and $W$ are in general position. Then $K \bmod W$ is a separation subdivision of $K$ if and only if the following two conditions are satisfied:

(3.13.1) If $\tau \in K_{2}$ and $J$ is a (nonempty) component of $W \cap|\tau|$, then $J$ separates $\tau$.

(3.13.2) If $\sigma \in K_{3}$ and $Y$ is a (nonempty) component of $W \cap \operatorname{Int} \sigma$, then $\mathrm{Cl} Y$ separates $\sigma$.

Proof. First check that $K \bmod W$ is a separation complex. Conditions (3.8.1) and (3.8.2) follow respectively from (3.13.1) and (3.13.2).

Lemma 3.14. Suppose the following are given:

(3.14.1) $Q$ is a $P L$ 2-sphere in $S^{3}$.

(3.14.2) $U$ is a component of $S^{3}-Q$.

(3.14.3) $K$ is a separation complex such that $\left|K_{1}\right| \subset Q$ and $\cap\left\{\right.$ Int $\left.\sigma \mid \sigma \in K_{3}\right\} \subset U$.

(3.14.4) $W$ is a compact PL 2-manifold-with-boundary such that $W \subset U$ and $W$ and $K$ are in general position (3.12).

Then $K \bmod W$ is a separation subdivision of $K$ if condition (3.13.1) is satisfied.

Proof. We check that (3.13.2) is also satisfied. Let $\sigma \in K_{3}, Y$ be a nonempty component of $W \cap \operatorname{Int} \sigma$, and $J$ be a nonempty component of Bd $Y$. By (3.13.1) $J$ separates the member $\tau$ of $K_{2}$ that includes $J$. Thus, let $\tau(J)$ be the closure of a component of $\tau-J$. By (3.14.3), $(\operatorname{Bd} \tau(J))-J \subset Q$. For each component $K$ of $(\operatorname{Bd} \tau(J))-J$ we add to $\tau(J)$ a disk in $Q$ bounded by $K$, thus we obtain a singular 2manifold $M(J)$ with boundary $J$. By (3.14.3), $M(J) \cap$ Int $\sigma=\varnothing$ for each component $J$ of $\mathrm{Bd} Y$. Thus, since the singular 2-manifold $(\cup\{M(J) \mid J$ is a component of $\mathrm{Bd} Y\}) \cup Y$ separates Int $\sigma, Y$ separates Int $\sigma$.

4. Winding functions. In this section we discuss the concept of a winding function which is used extensively in $\S \S 5$ and 6 . We begin by introducding notation that we use later for labeling abstract trees.

Definition 4.1. A tree labeling system is a collection $\Gamma$ of finite sequences $\left(a_{1}, a_{2}, \cdots, a_{n}\right)$ of nonnegative integers such that 
(4.1.1) $(0) \in \Gamma$ and is the only one element sequence in $\Gamma$,

(4.1.2) if $\left(a_{1}, a_{2}, \cdots, a_{n}\right) \in \Gamma$ then $\left(a_{1}, a_{2}, \cdots, a_{n-1}\right) \in \Gamma$ if $n \neq 1$, and $\left(a_{1}, a_{2}, \cdots a_{n-1}, a_{n}-1\right) \in \Gamma$ if $a_{n} \neq 0$.

(4.1.3) A maximal element of $\Gamma$ is finite sequence $\left(a_{1}, a_{2}, \cdots, a_{n}\right) \in$ $\Gamma$ such that there does not exist a nonnegative integer a such that $\left(a_{1}, a_{2}, \cdots a_{n}, a\right) \in \Gamma$.

(4.1.4) the lexicographical ordering of $\Gamma$ is $0,00,01,02, \cdots, 000$, $001, \cdots$, etc.

(4.1.5) a tree labeling system for set $B$ is a one-to-one function from $\Gamma$ onto $B$.

Notation. If $\Gamma$ is a tree labeling system for set $B$ then we use lower case greek letters to refer to elements of $\Gamma$ and denote the image of $\alpha \in \Gamma$ in $B$ by $b_{\alpha} \in B$. If $\alpha=\left(a_{1}, a_{2} \cdots, a_{n}\right) \in \Gamma$ and $\left(i_{1}, \cdots, i_{j}\right)$ is a sequence of nonnegative integers such that $\left(a_{1}, \cdots, a_{n}, i_{1}, \cdots, i_{j}\right) \in \Gamma$ then we write $a_{1} a_{2} \cdots a_{n} \in \Gamma, \alpha i_{1} i_{2} \cdots i_{j} \in \Gamma$, respectively. If $\alpha=$ $\left(a_{1}, a_{2}, \cdots, a_{n}\right) \in \Gamma$ then $|\alpha|=0$ if $n$ is an even integer and $|\alpha|=1$ if $n$ is an odd integer.

In items (4.2) through (4.9), $R$ is a $P L$ 2-sphere in $S^{3}, H$ is a disk-with-holes in $R, V$ is a component of $S^{3}-R$, and $M=h(H \times I)$ is a collar of $H$ in $\mathrm{Cl} V$.

Definition 4.2. A corner of $M$ is $h(E \times[0, t])$ where $E$ is a collar of $\mathrm{Bd} H$ in $H$ and $0<t<1$.

Definition 4.3. A compact, orientable, $P L$ 2-manifold $S$ in $M$ partitions $M$ if for each component $Y$ of $S$ we have $Y \cap \operatorname{Bd} M=$ $Y \cap((\mathrm{Bd} M)-H)=\mathrm{Bd} Y \neq \varnothing$.

The next lemma shows that a partition of $M$ induces a special type of separation subdivision of the separation complex $\bar{M}=\{J \mid J$ is a component $\mathrm{Bd} H\} \cup\{H, \mathrm{Bd} M$ - Int $H\} \cup\{M\}$.

Lemma 4.4. If $S$ partitions $M$ then $\bar{M} \bmod S=K_{1} \cup K_{2} \cup K_{3}$ is a separation complex having the property that the intersection of each pair of elements of $K_{3}$ is either empty or a single component of S. Furthermore, there exists a tree labeling system $\Gamma$ for $K_{3}$ such that $H \subset \mathrm{Bd} \sigma_{0}$ and $\sigma_{\alpha} \cap \sigma_{\beta}$ is nonempty if and only if either $\beta=\alpha i$ or $\alpha=\beta i$ for some nonnegative integer $i$.

Proof. Let $Y$ be component of $S$ and $J$ a component of $\operatorname{Bd} Y$. Since $\mathrm{Bd} M$ - Int $H$ is a disk-with-holes and $J \subset(\mathrm{Bd} M)-H, J$ separates $\mathrm{Bd} M$ - Int $H$. Thus, by Lemma $3.14, \bar{M} \bmod S$ is a separation complex. The other properties of $\bar{M} \bmod S$ follow by induction on the number of components of $S$. 
Definition 4.5. If $S$ partitions $M$ then we call $\bar{M} \bmod S$ the separation complex supported by $S$ in $M$.

Definition 4.6. If $R$ is a $P L$ 2-sphere in $S^{3}, V$ is a component of $S^{3}-R, H$ is a disk-with-holes in $R, M$ is a collar of $H$ in $\mathrm{Cl} V, S$ partitions $M$, and $K=K_{1} \cup K_{2} \cup K_{3}$ is the separation complex supported by $S$ in $M$ then function $P: M \rightarrow S^{3}$ is a winding function with respect to $(M, S, H, R)$ if

(4.6.1) $P \mid H=1$ and $P$ is the identity on a corner of $M$,

(4.6.2) if $\sigma \in K$ then $\{P(\tau) \mid \tau \in \bar{\sigma}\}=\overline{P(\sigma)}$ is a separation complex and $P \mid \sigma$ is a separation isomorphism from $\bar{\sigma}$ to $\overline{P(\sigma)}$,

(4.6.3) if $\sigma \in K_{3}$ then $P(\sigma-(S \cup H)) \subset S^{3}-R$ and $P(\sigma \cap(S \cup H)) \subset$ $R$,

(4.6.4) if $\sigma_{0}, \sigma_{1} \in K_{3}$ and $\sigma_{0} \cap \sigma_{1}$ is a component of $S$ then $P\left(\sigma_{0}-\right.$ $(S \cup H)), P\left(\sigma_{1}-(S \cup H)\right)$ lie in different components of $S^{3}-R$, and

(4.6.5) if $\sigma_{0}, \sigma_{1} \in K_{3}, \sigma_{0} \neq \sigma_{1}$, but $P\left(\sigma_{0}-(S \cup H)\right) \cap P\left(\sigma_{1}-(S \cup H)\right) \neq$ $\varnothing$ then for $i=0$, or $1, P\left(\sigma_{i}-(S \cup H)\right) \subset \operatorname{Int} P\left(\sigma_{1-i}\right)$ and $P\left(\sigma_{i} \cap(S \cup H)\right) \subset \operatorname{Int} P\left(\sigma_{1-i} \cap(S \cup H)\right)$.

We now establish several properties of a winding function.

LEMMA 4.7. If $P: M \rightarrow S^{3}$ is a winding function with respect to $(M, S, H, R), K=K_{1} \cup K_{2} \cup K_{3}$ is the separation complex supported by $S$ in $M$, and $\Gamma$ is a tree labeling system for $K_{3}$ satisfying Lemma 4.4. Then it is impossible to have $P\left(\sigma_{\beta}\right) \subset P\left(\sigma_{\alpha}\right)$ where $\alpha=\beta i_{1} \cdots i_{n}$ for some positive integer $n$. In particular, $P\left(\sigma_{0}\right) \subset P\left(\sigma_{\alpha}\right)$ implies $\alpha=0$.

Proof. Suppose the contrary, then there exist a smallest positive integer $n$ with the property that there exist $\alpha, \beta \in \Gamma$ such that $\alpha=$ $\beta i_{1} \cdots i_{n}$ and $P\left(\sigma_{\beta}\right) \subset P\left(\sigma_{\alpha}\right)$. By (4.6.3), (4.6.4), and (4.6.5) either $P\left(\sigma_{\beta i_{1}}\right) \subset P\left(\sigma_{\beta i_{1} \cdots i_{n-1}}\right)$ or there exists a nonnegative integer $i_{n+1}$ such that $P\left(\sigma_{\beta i_{1}}\right) \subset P\left(\sigma_{\beta i_{1} \cdots i_{n} i_{n+1}}\right)$. Suppose $P\left(\sigma_{\beta i_{1}}\right) \subset P\left(\sigma_{\beta i_{1} \cdots i_{n-1}}\right)$ then since $n$ is minimal, we must have $n=2$ and $\sigma_{\beta i_{1}}=\sigma_{\beta i_{1} \cdots i_{n-1}}$. But by (4.6.5) $P\left(\sigma_{\beta} \cap \sigma_{\beta i_{1}}\right) \subset \operatorname{Int} P\left(\sigma_{\beta i_{1} i_{2}} \cap \sigma_{\beta i_{1}}\right)$, thus we have distinct 2-faces of $\sigma_{\beta i_{1}}$ pinched together by $P$ contradicting (4.6.2). Hence we must have $P\left(\sigma_{\beta i_{1}}\right) \subset P\left(\sigma_{\beta i_{1} \cdots i_{n+1}}\right)$.

The above argument is repeated to $P\left(\sigma_{\beta i_{1}}\right) \subset P\left(\sigma_{\beta i_{1} \cdots i_{n+1}}\right)$ to find a nonnegative integer $i_{n+2}$ such that $P\left(\sigma_{\beta i_{1} i_{2}}\right) \subset P\left(\sigma_{\beta i_{1} \cdots i_{n+1} i_{n+2}}\right)$, and then repeated over again. Eventually a nonnegative integer $i_{n+p}$ is found such that $P\left(\sigma_{\beta i_{1} i_{2} \cdots i_{p}}\right) \subset P\left(\sigma_{\beta i_{1} \cdot \cdots i_{n+p}}\right)$ and $\beta i_{1} \cdots i_{p+n}$ is maximal in $\Gamma$. One more application of the argument yields a contradiction.

LEMma 4.8. If $P$ is a winding function with respect to ( $M, S$, $H, R)$ then 
(4.8.1) $\quad H-P(M-H)$ separates $P(M)-P(\operatorname{Bd} M)$ from $S^{3}-P(M)$ in $S^{3}-P(\operatorname{Bd} M-H)$, and

(4.8.2) $P(M)$ contains a collar of $P(\mathrm{Bd} M-\operatorname{Int} H)$ to the side of $P(\operatorname{Bd} M-\operatorname{Int} H)$ that contains a corner of $M$.

Proof. Suppose $p q$ is an arc from $p \in S^{3}-P(M)$ to $q \in P(M)-$ $P(\mathrm{Bd} M)$ that lies in $S^{3}-P(\operatorname{Bd} M-H)$. Let $r$ be the first point of $p q$ that intersect the compact set $P(M)$. Since $r$ is a boundary point of the 3-manifold $P(\sigma)$ for some $\sigma \in K_{3}$ and $p q \cap P(\operatorname{Bd} M-H)=\varnothing$ it follows that $r \in \operatorname{Int} P(S \cup H) \subset R$. By (4.6.2) and (4.6.4) since $r$ is the first point of intersection with $P(M), r \notin P(S)$. Hence $r \in P(H)-$ $P(S)=H-P(M-H)$ by (4.6.1) and (4.6.3), and the proof of (4.8.1) is complete.

Let $\Gamma$ be the tree labeling system for $K_{3}$ in Lemma 4.4. The collar required in (4.8.2) is constructed in $P(M)$ by induction on the elements of $\Gamma$. The set $P\left(\sigma_{0}\right)-\left(\cup P\left(\sigma_{0 \alpha}\right)\right)$ contains a collar $N_{0}$ of $\mathrm{Cl}\left(\left(\mathrm{Bd} P\left(\sigma_{0}\right)\right)-R\right)$ in $P\left(\sigma_{0}\right)$ such that $N_{0}$ contains a corner of $M$ by (4.6.1), (4.6.2) and Lemma 4.7. We may assume that $N_{0} \cap P\left(\sigma_{0} \cap S\right)$ is a collar of $\mathrm{Bd} P\left(\sigma_{0} \cap S\right)$ in $P\left(\sigma_{0} \cap S\right)$. Let $N_{0 i}$ be a collar of $\mathrm{Cl}\left(\left(\mathrm{Bd} P\left(\sigma_{0 i}\right)\right)-R\right)$ in $P\left(\sigma_{0 i}\right)-\left(\bigcup_{\alpha \in \Gamma_{0 i}} P\left(\sigma_{\alpha}\right)\right)$ where $\alpha \in \Gamma_{0 i}$ if and only if $P\left(\sigma_{\alpha}\right)-R \subset \operatorname{Int} P\left(\sigma_{0 i}\right)$ by (4.6.2) and (4.6.5). We may assume that $N_{0 i} \cap P\left(\sigma_{0 i} \cap S\right)$ is a collar of $\operatorname{Bd} P\left(\sigma_{0 i} \cap S\right)$ in $P\left(\sigma_{0} \cap S\right)$ and $N_{0} \cap P\left(\sigma_{0} \cap \sigma_{0 i}\right)=N_{0 i} \cap P\left(\sigma_{0} \cap \sigma_{0 i}\right)$. The induction step from $\sigma_{\alpha}$ to $\sigma_{\alpha i}$ is similar. The required collar is $\cup\left\{N_{\alpha} \mid \alpha \in \Gamma\right\}$.

The next lemma gives a lifting property for winding functions.

Lemma 4.9. If $P: M \rightarrow S^{3}$ is a winding function with respect to $(M, S, H, R), W$ is a compact 2-manifold such that each component of $W$ has nonempty boundary, $W$ is in general position with respect to $R$, Int $W \subset P(M)-P(\operatorname{Bd} M$ - Int $H)$, and $\mathrm{Bd} W \subset P(H)-P(S)$. Then there exists a compact $P L$ 2-manifold $\bar{W}$ in $M$ and a function $P^{\prime}: M \rightarrow$ $S^{3}$ such that

(4.9.1) $P^{\prime}|H=P| H=1$ and if $K=K_{1} \cup K_{2} \cup K_{3}$ is the separation complex supported by $S$ in $M$ then $P^{\prime}(\sigma)=P(\sigma)$ if $\sigma \in K$,

(4.9.2) $\bar{W}$ and $K$ are in general position and if $\bar{W}_{0}$ is a component of $\bar{W}$ then $\bar{W}_{0} \cap \operatorname{Bd} M=\bar{W}_{0} \cap H=\operatorname{Bd} \bar{W}_{0} \neq \varnothing$,

(4.9.3) $K^{\prime}=K \bmod \bar{W}=K_{1}^{\prime} \cup K_{2}^{\prime} \cup K_{3}^{\prime}$ is a separation subdivision of $K$,

(4.9.4) if $\sigma \in K_{3}$ then $P^{\prime} \mid \sigma$ is a separation isomorphism from $\bar{\sigma}^{\prime}$ to $\left\{P^{\prime}(\tau) \mid \tau \in \bar{\sigma}^{\prime}\right\}$,

(4.9.5) $\quad P^{\prime} \mid \bar{W}$ is a separation isomorphism from $\bar{W} \bmod (S \cup H)$ to $W \bmod R$,

(4.9.6) if $\sigma_{0}, \sigma_{1} \in K_{3}$ and $\sigma_{0} \neq \sigma_{1}$ but $P\left(\sigma_{0}\right) \subset P\left(\sigma_{1}\right)$ then there exists $\sigma_{2} \in K_{3}^{\prime}$ such that $\sigma_{2} \subset \sigma_{1}$ and $P\left(\sigma_{0}\right) \subset P^{\prime}\left(\sigma_{2}\right)$. 
Proof. Let $\Gamma$ be the tree labeling system for $K_{3}$ of Lemma 4.4. For $\sigma \in K_{3}$ let $\Gamma(\sigma)=\left\{\alpha \in \Gamma \mid P\left(\sigma_{\alpha}\right)\right.$ is a proper subset of $\left.P(\sigma)\right\}$ and let $W(\sigma)=W \cap\left(P(\sigma)-\cup\left\{P\left(\sigma_{\alpha}\right) \mid \alpha \in \Gamma(\sigma)\right\}\right)$. It is clear that $W=$ $\cup\left\{W(\sigma) \mid \sigma \in K_{3}\right\}$ and $W\left(\sigma_{\alpha}\right) \cap W\left(\sigma_{\beta}\right)=\varnothing$ if $\sigma_{\alpha} \cap \sigma_{\beta}=\varnothing$ by (4.6.5). Also, $W\left(\sigma_{\alpha}\right) \cap W\left(\sigma_{\alpha i}\right)=W \cap\left(P\left(\sigma_{\alpha} \cap \sigma_{\alpha i}\right)-\cup\left\{P\left(\sigma_{\beta}\right) \mid \beta \in \Gamma\left(\sigma_{\alpha}\right)\right\}\right)=W \cap$ $\left(P\left(\sigma_{\alpha} \cap \sigma_{\alpha i}\right)-\cup\left\{P\left(\sigma_{\beta}\right) \mid \beta \in \Gamma\left(\sigma_{\alpha i}\right)\right\}\right)$ by (4.6.4) and the fact that $W$ is in general position with respect to $R$. By hypothesis on $W$, each component $W_{i}(\sigma)$ of $W(\sigma)$ is a compact 2-manifold with $\varnothing \neq \mathrm{Bd} W_{i}(\sigma) \subset$ Int $P(\sigma \cap(S \cup H)) \subset R$. The 2-manifold $W_{i}(\sigma)$ separates the 3-cell bounded by $R$ and containing $W_{i}(\sigma)$ : consequently, $W_{i}(\sigma)$ separates $P(\sigma)$. If $t$ is a boundary component of $W_{i}(\sigma)$ then there exists a component $Y$ of $\sigma \cap(S \cup H)$ such that $t \subset \operatorname{Int} P(Y) \subset R$. Since $P(Y)$ is a disk-with-holes, $t$ separates $P(Y)$. It now follows by Lemma 3.13 that $L(\sigma)=L_{1}(\sigma) \cup L_{2}(\sigma) \cup L_{3}(\sigma)=\overline{P(\sigma)} \bmod W(\sigma)$ is a separation subdivision of $\{P(\tau) \mid \tau \in \bar{\sigma}\}=\overline{P(\sigma)}$.

We use Lemma 3.10 to lift the 'separation subdivision $L(\sigma)$ of $\overline{P(\sigma)}$ to $\bar{\sigma}$. By Lemma 3.10 there exists a subdivision $\bar{\sigma}_{0}^{\prime}$ of $\bar{\sigma}_{0}$ and a separation isomorphism $P^{\prime} \mid \sigma_{0}$ from $\bar{\sigma}_{0}^{\prime}$ to $L\left(\sigma_{0}\right)$ such that $\left(P^{\prime} \mid \sigma_{0}\right)(\tau)=$ $P(\tau)$ if $\tau \in \bar{\sigma}_{0}$, and $\left(P^{\prime} \mid \sigma_{0}\right)|H=P| H$. Proceeding inductively, we assume that separation subdivision $\bar{\sigma}_{\alpha}^{\prime}$ of $\bar{\sigma}_{\alpha}$ and separation isomorphism $P^{\prime} \mid \sigma_{\alpha}$ from $\bar{\sigma}_{\alpha}^{\prime}$ to $L\left(\sigma_{\alpha}\right)$ have been defined such that $\left(P^{\prime} \mid \sigma_{\alpha}\right)(\tau)=P(\tau)$ if $\tau \in \bar{\sigma}_{\alpha}$. Let $i$ be a nonnegative integer such that $\alpha i \in \Gamma$, as we have observed before $W \cap\left(P\left(\sigma_{\alpha} \cap \sigma_{\alpha i}\right)-\cup\left\{P\left(\sigma_{\beta}\right) \mid \beta \in \Gamma\left(\sigma_{\alpha}\right)\right\}=W \cap\left(P\left(\sigma_{\alpha} \cap \sigma_{\alpha i}\right)-\right.\right.$ $\left.\cup\left\{P\left(\sigma_{\beta}\right) \mid \beta \in \Gamma\left(\sigma_{\alpha i}\right)\right\}\right)$ so by Lemma 3.10 there exists a separation subdivision $\bar{\sigma}_{\alpha i}^{\prime}$ of $\bar{\sigma}_{\alpha i}$ and a separation isomorphism $P^{\prime} \mid \sigma_{\alpha i}$ from $\bar{\sigma}_{\alpha i}^{\prime}$ to $L\left(\sigma_{\alpha i}\right)$ such that $\left(P^{\prime} \mid \sigma_{\alpha i}\right)(\tau)=P(\tau)$ if $\tau \in \bar{\sigma}_{\alpha i}$ and $\left(P^{\prime} \mid \sigma_{\alpha i}\right) \mid \sigma_{\alpha} \cap \sigma_{\alpha i}=$ $\left(P^{\prime} \mid \sigma_{\alpha}\right) \mid \sigma_{\alpha} \cap \sigma_{\alpha i}$.

Let $P^{\prime}=\bigcup_{\alpha \in K_{3}}\left(P^{\prime} \mid \sigma\right), \bar{W}=\bigcup_{\alpha \in K_{3}}\left(P^{\prime} \mid \sigma\right)^{-1}(W(\sigma))$, and $K^{\prime}=\bigcup_{\alpha \in K_{3}} \bar{\sigma}^{\prime}$. Properties (4.9.1), $\cdots,(4.9 .5)$ are straight forward consequences of the construction. Property (4.9.6) follows since $P\left(\sigma_{0}\right) \subset P\left(\sigma_{1}\right)$ implies that $P\left(\sigma_{0}\right) \cap W\left(\sigma_{1}\right)=\varnothing$ and the latter implies $P\left(\sigma_{0}\right)$ is included in an element of $L_{3}\left(\sigma_{1}\right)$ since $P\left(\sigma_{0}\right)$ is connected.

5. Structure theorems. The purpose of this section is to prove the combinatorial structure theorems (Lemma 5.15 and Theorem 5.16) for components associated with the general position of two arbitrary $P L 2$-spheres in $E^{3}$. Throughout this section an arbitrary $P L 2$-sphere $R$ in $S^{3}$ is fixed. Also, a choice function $\mu$ on the collection of nonempty sets of simple closed curves in $R$ is fixed. Some of the objects defined below depend on $R$ or $\mu$, but to save notation no special attention is called to this fact.

Definition 5.1. Let $\mathscr{S}$ be the collection of all 4-tuples $(X, U$, $q, m)$ such that 
(5.1.1) $X$ is a compact, connected, $P L$ 2-manifold in $S^{3}$ without boundary and in general position with respect to $R$ such that if $t$ is a component (simple closed curve) of $R \cap X$ then $t$ separates $X$,

(5.1.2) $\mathrm{U}$ is one of the two components of $S^{3}-X$,

(5.1.3) $q$ is a point of $X-R$, and

(5.1.4) $m$ is a component (simple closed curve) of $R \cap X$.

For $a=(X, U, q, m) \in \mathscr{S}$ the existence of the following objects is clear and we use the following notation.

(5.2) $A_{a}$ is the closure of the component of $X-m$ that does not contain $q$,

(5.3) $\quad V_{a}$ is the component of $S^{3}-R$ that contains the interior of a collar of $m$ in $A_{a}$,

(5.4) $B_{a}$ is the disk in $R$ bounded by $m$ that contains a collar of $m$ in $R \cap \mathrm{Cl} U$,

(5.5) $C_{a}$ is the closure of the component of $A_{a}-R$ that contains $m$ in its boundary,

(5.6) $D_{a}$ is the closure of the component of $B_{a}-C_{a}$ that contains $m$ in its boundary,

(5.7) $|a|$ is the number of components of $A_{a}-R$, and

(5.8) $L_{a}$ is the collection of all components $t$ of $\mathrm{Bd} C_{a}-\mathrm{Bd} D_{a}$ such that $B_{b} \cap D_{a}=\varnothing$ where $b=(X, U, q, t)$.

Since $C_{a}$ separates $\mathrm{Cl} V_{a}$ and $R$ is a 2-sphere, the following lemma is easily established.

Lemma 5.9 The sets $C_{a}, D_{a}$, and $L_{a}$ have the following properties:

(5.9.1) $\operatorname{Bd} D_{a} \subset \operatorname{Bd} C_{a}$,

(5.9.2) there exists a collar of $\mathrm{Bd} D_{a}$ in $D_{a} \cap \mathrm{Cl} U$, and

(5.9.3) if $\mathrm{Bd} C_{a}-\operatorname{Bd} D_{a} \neq \varnothing$ then $L_{a} \neq \varnothing$.

The existence of the following objects associated with $a=$ $(X, U, q, m) \in \mathscr{S}$ will be established by induction on $|a|$ in Lemma 5.15. See Figure 2.

(5.10a) $H_{a}$ is a disk-with-holes in $D_{a}$ such that

(5.10.1a) $m \subset \mathrm{Bd} H_{a}$, and $\mathrm{Bd} H_{a} \subset X \cap R$,

(5.10.2a) there exists a collar of $\mathrm{Bd} H_{a}$ in $H_{a} \cap \mathrm{Cl} U$,

(5.10.3a) $\mathrm{Bd} H_{a}$ is the boundary of a compact, connected, 2manifold $J_{a}$ in $A_{a}$.

(5.11a) $\quad M_{a}=h\left(H_{a} \times I\right)$ is a collar of $H_{a}$ in $\mathrm{Cl} V_{a}$ such that

(5.11.1a) $h\left(\mathrm{Bd} H_{a} \times I\right)$ is a collar of $\mathrm{Bd} J_{a}$ in $J_{a}$,

(5.11.2a) $M_{a}$ intersects a component $Y$ of $X \cap V_{a}$ if and only if $\mathrm{Cl} Y$ intersects $H_{a}$,

(5.12a) $S_{a}$ is a partition of $M_{a}$ and $K_{a}=K_{1}(a) \cup K_{2}(a) \cup K_{3}(a)$ is the separation complex supported by $S_{a}$ in $M_{a}$, 


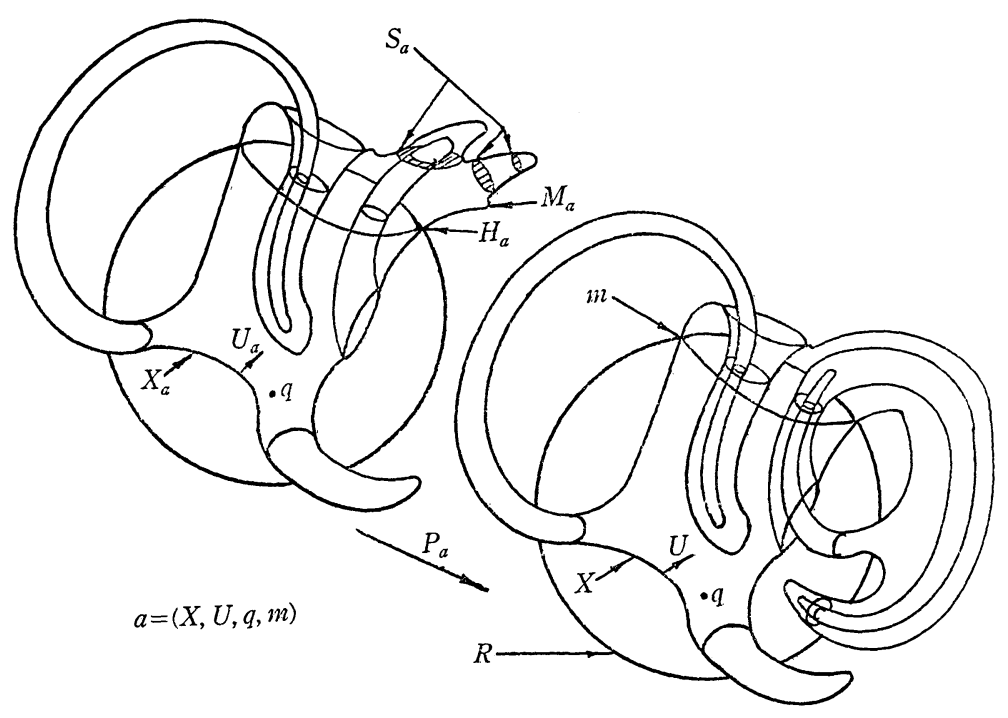

FIGURE 2

(5.13a) $X_{a}$ is compact, connected, $P L$ 2-manifold in $S^{3}$ without boundary and in general position with respect to $R \cup S_{a}$ such that

(5.13.1a) $X_{a}$ contains $G_{a}=\left(\mathrm{Bd} M_{a}\right)-$ Int $H_{a}$,

(5.13.2a) if $t$ is a component of $\left(R \cup S_{a}\right) \cap X_{a}$ then $t$ separates $X_{a}$,

(5.13.3a) if $t$ is a component of $\left(X_{a}-G_{a}\right) \cap S_{a}$ then $t$ separates the component of $S_{a}$ containing $t$.

From (5.13.3a), the fact that $\left(R-H_{a}\right) \cup G_{a}$ is a 2-sphere, and Lemma 3.14 it follows that $K_{a}^{\prime}=K_{a} \bmod \left(X_{a}-\operatorname{Int} G_{a}\right)=K_{1}^{\prime}(a) \cup K_{2}^{\prime}(a) \cup$ $K_{3}^{\prime}(a)$ is a separation subdivision of $K_{a}$.

(5.14a) $P_{a}$ is a function from $S^{3}$ to $S^{3}$ such that

(5.14.1a) $P_{a} \mid S^{3}-\left(M_{a}-H_{a}\right)=1$,

(5.14.2a) $P_{a} \mid M_{a}$ is a winding function with respect to $\left(M_{a}, S_{a}\right.$, $\left.H_{a}, R\right)$,

(5.14.3a) $P_{a} \mid X_{a}$ is a separation isomorphism from $\bar{X}_{a}=X_{a}$ $\bmod \left(R \cup S_{a}\right)$ to $\bar{X}=X \bmod R$.

(5.14.4a) $\quad P_{a}\left(G_{a}\right)=J_{a}$,

(5.14.5a) $\quad P_{a}\left(M_{a}\right) \cap\left(X_{a}-M_{a}\right)=\varnothing$,

(5.14.6a) if $\sigma \in K_{3}(a)$ then $P_{a} \mid \sigma$ is a separation isomorphism from $\bar{\sigma}^{\prime}$ to $\left\{P_{a}(\tau) \mid \tau \in \bar{\sigma}^{\prime}\right\}$, and

(5.14.7a) if $\sigma_{0}, \sigma_{1} \in K_{3}(\alpha)$ and $\sigma_{0} \neq \sigma_{1}$ but $P_{a}\left(\sigma_{0}\right) \subset P_{a}\left(\sigma_{1}\right)$ then there exists $\sigma_{2} \in K_{3}^{\prime}(a)$ such that $\sigma_{2} \subset \sigma_{1}$ and $P_{a}\left(\sigma_{0}\right) \subset$ $P_{a}\left(\sigma_{2}\right)$.

Lemma 5.15. If $a=(X, U, q, m) \in \mathscr{S}$ where $\mathscr{S}$ is given by (5.1) 
then there exists a 5-tuple $\left(H_{a}, M_{a}, S_{a}, X_{a}, P_{a}\right)$ that satisfies (5.10a), (5.11a), $\cdots$, , (5.14a).

Proof. The proof is by induction on $|a|$ given by (5.7). We use the notation given by $(5.1), \cdots,(5.8)$.

Suppose $a=(X, U, q, m) \in \mathscr{S}$ and $|a|=1$. It is clear that $C_{a}=$ $A_{a}, D_{a}=B_{a}$ and $\mathrm{Bd} C_{a}=\operatorname{Bd} D_{a}$. Let $H_{a}=D_{a}, J_{a}=C_{a}$ and let $h$ be a $P L$ embedding of $H_{a} \times I$ into $\mathrm{Cl} V_{a}$ such that $h(y \times 0)=y, h\left(H_{a} \times\right.$ $(0,1]) \subset V_{a}, h\left(\mathrm{Bd} H_{a} \times I\right)$ is a collar of $\mathrm{Bd} J_{a}$ in $J_{a}$, and $h\left(H_{a} \times I\right)$ intersects a component $Y$ of $X \cap V_{a}$ if and only if Cl $Y$ intersects $H_{a}$. Let $M_{a}=h\left(H_{a} \times I\right)$ and let $S_{a}=\varnothing$. A one-to-one function $P$, given by $P \mid H_{a}=1, P\left(G_{a}\right)=J_{a}, P\left(\operatorname{Int} M_{a}\right)=\operatorname{Int} L$ where $L$ is the 3 -manifold bounded by $H_{a} \cup J_{a}$ and containing a collar of $J_{a}$ in $\mathrm{Cl} U$, is a trivial winding function with respect to $\left(M_{a}, S_{a}, H_{a}, R\right)$. Let $W=X \cap\left(L-J_{a}\right)$ and apply Lemma 4.9 to $P$ and $W$ and let $X_{a}=\bar{W} \cup(X-L) \cup G_{a}$, $P_{a} \mid M_{a}=P^{\prime}$ where $\bar{W}, P^{\prime}$ are as in the conclusion of Lemma 4.9. It is straight forward to check that $\left(H_{a}, M_{a}, S_{a}, X_{a}, P_{a}\right)$ satisfies (5.10a), $\cdots,(5.14 \mathrm{a})$.

Inductively, we suppose that the 5-tuple $\left(H_{b}, M_{b}, S_{b}, X_{b}, P_{b}\right)$ exists with properties $(5.10 \mathrm{~b}), \cdots,(5.14 \mathrm{~b})$ provided that $|b|<k$. Let $a=$ $(X, U, q, m) \in \mathscr{S}$ with $|a|=k$. By Lemma 5.9.1 we have that $\operatorname{Bd} D_{a}=$ $\mathrm{Bd} C_{a}$ or $\mathrm{Bd} C_{a}-\mathrm{Bd} D_{a} \neq \varnothing$ and we accordingly, break the induction step into two cases.

Case A. $\mathrm{Bd} D_{a}=\mathrm{Bd} C_{a}$. In this case we do not need the induction hypothesis and we proceed formally as above for $|a|=1$. That is, we let $H_{a}=D_{a}, J_{a}=C_{a}, M_{a}$ be a collar of $H_{a}$ in $\mathrm{Cl} V_{a}, S_{a}=\varnothing, L$ be the 3-manifold bounded by $H_{a} \cup J_{a}$ and containing a collar of $J_{a}$ in $\mathrm{Cl} U$, and let $X_{a}, P_{a}$ be obtained by the same application of Lemma 4.9 as was used above.

Case B. $\mathrm{Bd} C_{a}-\mathrm{Bd} D_{a} \neq \varnothing$. See Figure 3. By Lemma 5.9.3, $L_{a} \neq \varnothing$ and we let $m_{a}=\mu\left(L_{a}\right)$ where $\mu$ is the choice function mentioned in the first paragraph of $\S 5$. Let $b=\left(X, U, q, m_{a}\right)$. Since $m_{a} \subset A_{a}$ we have that $A_{b} \subset A_{a}$ and $|b|<|a|=k$. By the induction hypothesis a 5-tuple $\left(H_{b}, M_{b}, S_{b}, X_{b}, P_{b}\right)$ exists with properties $(5.10 \mathrm{~b}), \cdots,(5.14 \mathrm{~b})$. By (5.6), $m \subset D_{a}$. by (5.10b), (5.6), (5.9), $H_{b} \subset D_{b} \subset B_{b}$ : and by (5.8) $B_{b} \cap D_{a}=\varnothing$. Hence, $D_{a} \cap H_{b}=\varnothing, m \cap H_{b}=\varnothing$ and $M_{b} \cap m=\varnothing$ by (5.11b). Also, by (5.14.1b) and $(5.14 .3 \mathrm{~b})$ we have that $m \subset X_{b}$. Let $g$ be a $P L$ homeomorphism of $S^{3}$ onto $S^{3}$ that is fixed outside a thin shell neighborhood of $M_{b}$ and pushes $M_{b}$ across $R$ to $S^{3}-\mathrm{Cl} V_{b}$. Since $D_{a} \cap H_{b}=\varnothing, m \cap H_{b}=\varnothing$ it follows that no point near $D_{a}$ is moved by $g$ and $g(m)=m$. Let $c=\left(g\left(X_{b}\right), g\left(U_{b}\right), g\left(q_{b}\right), m\right)$ where $U_{b}$ is the component of $S^{3}-X_{b}$ such that $H_{b} \cap \mathrm{Cl} U_{b}$ contains the collar of $\mathrm{Bd} H_{b}$ 


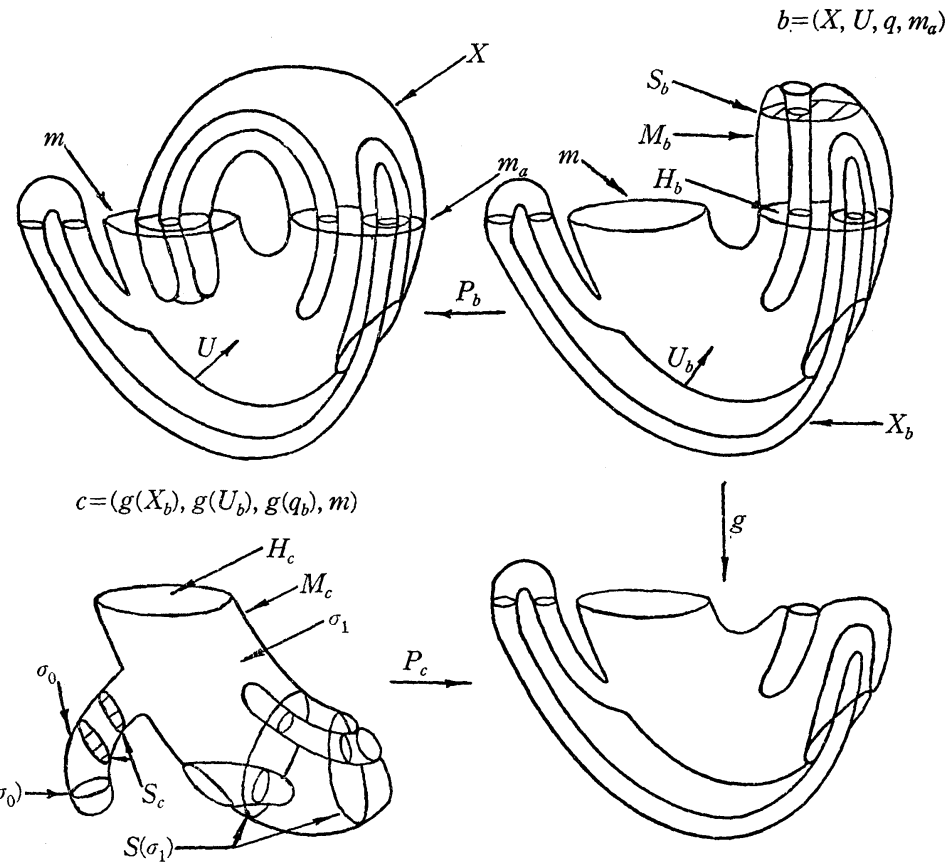

FiguRE 3

given in (5.10.2b), and $q_{b}=\left(P_{b} \mid X_{b}\right)^{-1}(q)$. Since $g$ removes components of $R \cap X_{b}$, in particular $m_{a}$, and by property $(5.14 .3 \mathrm{~b})$, it follows that $|c|<|b|<|a|=k$. By the induction hypothesis, a 5-tuple $\left(H_{c}, M_{c}\right.$, $\left.S_{c}, X_{c}, P_{c}\right)$ exists with properties $(5.10 \mathrm{c}), \cdots,(5.14 \mathrm{c})$.

Let $H_{a}=H_{c}, M_{a}=M_{c}$, and $J_{a}=P_{b} g^{-1}\left(J_{c}\right)$. By pushing $M_{a}$ into a smaller neighborhood of $H_{a}$ if necessary, we may assume that (5.11.2a) is satisfied and $g^{-1} \mid M_{a}=1$. Properties (5.10a), (5.10.1a), (5.10.2a), (5.10.3a), (5.11a), (5.11.1a) are consequences of the corresponding properties for $c$ and the facts that $P_{b} \mid M_{a}=1$ and $g^{-1} \mid M_{a}=1$.

It follows from (4.7) that $P_{c}\left(M_{c}\right)$ misses the interior of a collar of $m$ in $\mathrm{Cl}\left(S^{3}-V_{c}\right)$. Since $H_{b}$ is in the same component of $S^{3}-$ $P_{c}\left(\mathrm{Bd} M_{c}-H_{c}\right)$ that contains the interior of such a collar of $m$ in $\mathrm{Cl}\left(S^{3}-V_{c}\right)$, it follows from (4.8.1) and (4.7) that $H_{b} \cap P_{c}\left(M_{c}\right)=\varnothing$.

Let $\sigma \in K_{3}(c)$. Since $g^{-1}$ is a homeomorphism that only moves points near $H_{b}$ into $M_{b}$ and $H_{b} \cap P_{c}\left(M_{c}\right)=\varnothing$ we have by (5.14.2c), (4.6.2), (4.6.3) that $g^{-1} P_{c} \mid \sigma$ is a separation isomorphism from $\bar{\sigma}$ to $\left\{g^{-1} P_{c}(\tau) \mid \tau \in \bar{\sigma}\right\}=$ $\overline{g^{-1} P_{c}(\sigma)}$ such that $g^{-1} P_{c}\left(\sigma \cap S_{c}\right) \subset R-H_{b}$. Let $W=S_{b} \cup H_{b}$ and let $Q$ be the 2-sphere obtained from $\left(R-H_{b}\right) \cup G_{b}$ by pushing $G_{b}$ slightly away from $M_{b}$. Since $g^{-1} P_{c}\left((\mathrm{Bd} \sigma)-S_{c}\right) \subset X_{b}$ and $X_{b}$ satisfies $(5.13 \mathrm{~b})$, (5.13.2b), (5.13.3b) it follows that each component $t$ of $g^{-1} P_{c}((\mathrm{Bd} \sigma)-$ $\left.S_{c}\right) \cap W$ separates the component of $W$ containing $t$ or $t \subset \mathrm{Bd} W$, and $t$ separates the 2 -face of $\overline{g^{-1} P_{c}(\sigma)}$ containing $t$. By Lemma 3.14 applied to $Q$ and $W, \overline{g^{-1} P_{c}(\sigma)} \bmod W$ is a separation subdivision of 
$\overline{g^{-1} P_{c}(\sigma)}$. By Lemma 3.10 we assume without loss of generality that $S(\sigma)$ is a compact $P L$ 2-manifold in $\sigma$ such that if $Y$ is a component of $S(\sigma)$ then $Y \cap \mathrm{Bd} \sigma=Y \cap\left((\mathrm{Bd} \sigma)-S_{c}\right)=\mathrm{Bd} Y \neq \varnothing$ and $g^{-1} P_{c}(Y)$ is a component of $W \cap g^{-1} P_{c}(\sigma)$.

Let $S_{a}=S_{c} \cup\left(\cup\left\{S(\sigma) \mid \sigma \in K_{3}(c)\right\}\right)$. It is clear that $S_{a}$ satisfies (5.12) and $g^{-1} P_{c} \mid \sigma \in K_{3}(\alpha)$ is a separation isomorphism from $\bar{\sigma}$ to $\left\{g^{-1} P_{c}(\tau) \mid \tau \in \bar{\sigma}\right\}$ that satisfies property (4.6.5).

Let $P=P_{b} g^{-1} P_{c} \mid M_{a}$. We now show that $P$ is a winding function with respect to $\left(M_{a}, S_{a}, H_{a}, R\right)$. Property (4.6.1) is satisfied since $P_{c}$ satisfies (4.6.1), and $g^{-1}, P_{b}$, as pointed out previously, are the identity on a neighborhood of $H_{a}$. Property (4.6.2) is established by observing that, by the way $K_{a}$ was constructed in the previous two paragraphs, if $\sigma \in K_{3}(a)$ then $M_{b} \cap \operatorname{Int} g^{-1} P_{c}(\sigma)=\varnothing$ or $g^{-1} P_{c}(\sigma)=|L|$ where $L$ is a subcomplex of $\bar{\sigma}_{0}^{\prime}$ for some $\sigma_{0} \in K_{3}(b) \quad\left(\bar{\sigma}_{0}^{\prime}\right.$ is the induced subdivision of $\bar{\sigma}_{0}$ by $K_{b}^{\prime}$ ). In the former case, (4.6.2) follows since $P_{b} \mid S^{3}-\left(M_{b}-H_{b}\right)=1$. In the latter case, (4.6.2) follows by (5.14.6b). Properties (4.6.3) and (4.6.4) are easy consequences of the corresponding propertis for $P_{b}$ and $P_{c}$ and the way $S_{a}$ was constructed in the previous two paragraphs. We now establish property (4.6.5). Suppose $\sigma_{0}, \sigma_{1} \in K_{3}(a), \sigma_{0} \neq \sigma_{1}$ and $P\left(\sigma_{0}-S_{a}\right) \cap P\left(\sigma_{1}-S_{b}\right) \neq \varnothing$, then (a) $M_{b} \cap \operatorname{Int} g^{-1} P_{c}\left(\sigma_{i}\right)=\varnothing$ for $i=0,1$ or (b) $g^{-1} P_{c}\left(\sigma_{i}\right) \subset M_{b}, M_{b} \cap$ Int $g^{-1} P_{c}\left(\sigma_{1-i}\right)=\varnothing$ for $i=0,1$ or (c) $g^{-1} P_{c}\left(\sigma_{i}\right) \subset M_{b}, i=0,1$. In Case (a), (4.6.5) follows from the corresponding property for $P_{c}$ and the fact that $g^{-1}$ is a homeomorphism. In Case (b), $P\left(\sigma_{i}-S_{a}\right) \subset \operatorname{Int} P\left(\sigma_{1-i}\right)$ since $P\left(\sigma_{i}-S_{a}\right)$ is connected and fails to contain any point in $B \mathrm{~d} P\left(\sigma_{1-i}\right)$ by (5.14.5b). In Case (c), either (c.1) there exists a $\sigma^{*} \in K_{3}(b)$ such that $g^{-1} P_{c}\left(\sigma_{i}\right) \subset \sigma^{*}$ for $i=0,1$ or (c.2) there exists distinct $\sigma_{0}^{*}, \sigma_{1}^{*} \in$ $K_{3}(b)$ such that $g^{-1} P_{c}\left(\sigma_{i}\right) \subset \sigma_{1}^{*}$. In Case (c.1), (4.6.5) follows from the corresponding property for $P_{c}$ and the fact that $g^{-1}$ is a homeomorphism and (5.14.6b). In Case (c.2), (4.6.5) follows from (5.14.7b) since we must have $P_{b}\left(\sigma_{1}^{*}\right) \subset P_{b}\left(\sigma_{1-i}^{*}\right)$ for $i=0$ or 1 , by property (4.6.5) for $P_{b}$.

Let $W=X \cap P\left(M_{a}\right)-P\left(\mathrm{Bd} M_{a}\right.$ - Int $\left.\left.H_{a}\right)\right)$. Apply Lemma 4.9 to $P$ and $W$ to obtain $\bar{W}$ and $P^{\prime}$ satisfying (4.9.1), $\cdots,(4.9 .6)$.

Let $X_{a}=\bar{W} \cup G_{a} \cup\left(X-P^{\prime}\left(M_{a}\right)\right)$ and let $P_{a}$ be given by $P_{a} \mid M_{a}=$ $P^{\prime}$ and $P_{a} \mid S^{3}-\left(M_{a}-H_{a}\right)=1$. We now varify the remaining properties of the conclusion of the Lemma 5.15 to complete the induction step. Properties (5.14.1a), (5.14.2a) are clear by (4.9.1) applied to $P^{\prime}$. Property (5.14.3a) follows from (4.9.5) applied to $P^{\prime}$, the definition given previously of $J_{a}$, and the fact that $P \mid S^{3}-\left(M_{a}-H_{a}\right)=1$. Property (5.14.4a) is a consequence of the definition of $J_{a}$. Property (5.14.5a) follows since $X_{a}-M_{a}=X-P^{\prime}\left(M_{a}\right)$. Properties (5.14.6a) and (5.14.7a) are consequences of (4.9.4) and (4.9.6) respectively, applied to $P^{\prime}$. Properties (5.13.1a), (5.13.2a), and (5.13.3a) follow from the definition of $X_{a}$ and (4.9.3), (4.9.5). 


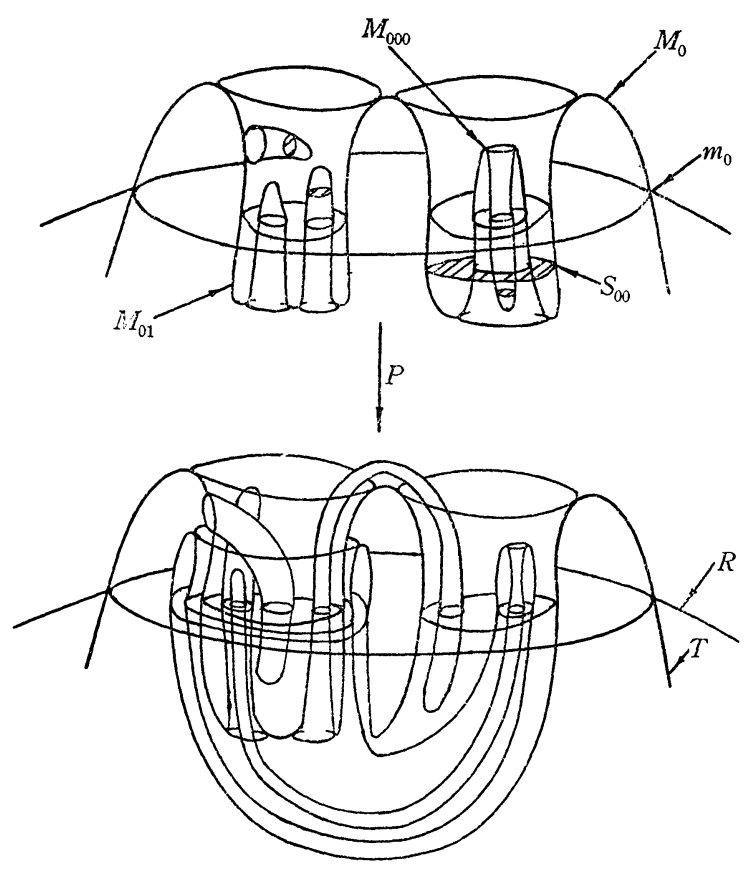

FIGURE 4

The following theorem gives the combinatorial structure of the collection of components of $R \cap T, R-T, T-R, S^{3}-(T \cup R)$ where $R$ and $T$ are $P L 2$-spheres in general position in $S^{3}$. The reader is referred to Definition 4.1 for some of the notation used. See Figure 4 for an illustration.

Theorem 5.16. Suppose $R, T$ are $P L$ 2-spheres in $S^{3}$ that are in general position and $m$ is a simple closed curve in $R \cap T$ such that $m$ bounds a disk $D$ in $T$ and $a$ disk $E$ in $R$ such that $R \cap T \subset D \cap E$. Let $B$ be the 3-cell bounded by $\mathrm{Cl}(T-D) \cup \mathrm{Cl}(R-E)$ such that Int $B \cap$ Int $D=$ Int $B \cap$ Int $E=\varnothing$ and let $U_{0}, U_{1}$ and $V_{0}, V_{1}$ be respectively the components of $S^{3}-T$, and $S^{3}-R$ and such that Int $B \subset U_{0}$, Int $\mathrm{B} \subset V_{0}$. Then there exists a finite tree labeling system $\Gamma$, a 4-tuple $\left(m_{\alpha}, H_{\alpha}\right.$, $M_{\alpha}, S_{\alpha}$ ) for each $\alpha \in \Gamma$, and a function $P$ from $S^{3}$ to $S^{3}$ such that

(5.16.1) $m_{\alpha}$ is a component (a simple closed curve) of $D \cap E$ with $m_{0}=m=\mathrm{Bd} D=\mathrm{Bd} E$,

(5.16.2) $\quad H_{\alpha}$ is a disk-with-holes in $E$ such that

(5.16.2.1) $\quad \mathrm{Bd} H_{\alpha}=m_{\alpha} \cup m_{\alpha 0} \cup m_{\alpha 1} \cup m_{\alpha 2} \cup \cdots$,

(5.16.2.2) $\quad E=\cup\left\{H_{\alpha} \mid \alpha \in \Gamma\right\}, H_{\alpha} \cap H_{\alpha i}=m_{\alpha i}$,

(5.16.2.3) $H_{\alpha}$ contains a collar of $\mathrm{Bd} H_{\alpha}$ in $\mathrm{Cl}\left(U_{|\alpha|}\right)$,

(5.16.3) $\quad M_{\alpha}$ is a collar of $H_{\alpha}$ in $\mathrm{Cl} V_{|\alpha|}$ such that if $|\alpha|=|\beta|$ and $\alpha \neq \beta$ then $M_{\alpha} \cap M_{\beta}=\varnothing$,

(5.16.4) $S_{\alpha}$ is a partition of $M_{\alpha}$ that supports the separation 
complex $K(\alpha)=K_{1}(\alpha) \cup K_{2}(\alpha) \cup K_{3}(\alpha)$, and if $D^{*}=\bigcup_{\alpha \in \Gamma}\left(\mathrm{Bd} M_{\alpha}-\operatorname{Int} H_{\alpha}\right), S=\bigcup_{\alpha \in \Gamma} S_{\alpha}$, and $K=K_{1} \cup K_{2} \cup K_{3}, \bar{D}^{*}$, $\bar{D}$ are the separation complexes given respectively by $K_{i}=\bigcup_{\alpha \in \Gamma} K_{i}(\alpha)$, $\bar{D}^{*}=D^{*} \bmod (S \cup E)$ and $\bar{D}=D \bmod E$ then

(5.16.5) $P \mid S^{3}-(|K|-E)=1$, and $P$ is the identity on a corner of $M_{\alpha}$ for each $\alpha \in \Gamma$,

(5.16.6) $P \mid D^{*}$ is a separation isomorphism from $\bar{D}^{*}$ to $\bar{D}$, and a homeomorphism from $D^{*}$ onto $D$,

(5.16.7) if $\sigma \in K$ then $\{P(\tau) \mid \tau \in \bar{\sigma}\}=\overline{P(\sigma)}$ is a separation complex and $P \mid \sigma$ is a separation isomorphism from $\bar{\sigma}$ to $\overline{P(\sigma)}$,

(5.16.8) if $\sigma \in K_{3}$ then $P(\sigma-(S \cup E)) \subset S^{3}-R$ and $P(\sigma \cap(S \cup E)) \subset E$,

(5.16.9) if $\sigma_{0}, \sigma_{1} \in K_{3}$ and $\sigma_{0} \cap \sigma_{1}$ is a component of $S$ then $P\left(\sigma_{0}-\right.$ $(S \cup E)), P\left(\sigma_{1}-(S \cup E)\right)$ lie in different components of $S^{3}-R$, and

(5.16.10) if $\sigma_{0}, \sigma_{1} \in K_{3}, \sigma_{0} \neq \sigma_{1}$, but $P\left(\sigma_{0}-(S \cup E)\right) \cap P\left(\sigma_{1}-(S \cup E)\right) \neq$ $\varnothing$ then for $i=0$, or $1, P\left(\sigma_{i}-(S \cup E)\right) \subset \operatorname{Int} P\left(\sigma_{1-i}\right)$ and $P\left(\sigma_{i} \cap(S \cup E)\right) \subset \operatorname{Int} P\left(\sigma_{1-i} \cap(S \cup E)\right)$.

Proof. The sets $m_{\alpha}, H_{\alpha}, M_{\alpha}, S_{\alpha}$, and function $P$ are constructed inductively using Lemma 5.15, Lemma 3.14 and the properties of winding functions given in (4.6), (4.7), (4.8), and (4.9). Let $q$ be a point in $T-D$ and identify the 2 -sphere $R$ of the hypothesis and the 2-sphere $R$ of $\S 5$.

The 4-tuple $a_{0}=\left(T, U_{1}, q, m\right)$ is in $\mathscr{S}$ of Definition 5.1. Let $\left(H_{0}, M_{0}, S_{0}, X_{0}, P_{0}\right)$ be the 5-tuple of the conclusion of Lemma 5.15 with $K(0)=K_{1}(0) \cup K_{2}(0) \cup K_{4}(0)$ the seperation complex supported by $S_{0}$ in $M_{0}$ with $K_{1}^{\prime}(0) \cup K_{2}^{\prime}(0) \cup K_{3}^{\prime}(0)$ the separation subdivision of $K(0)$ of Lemma 5.15. Let $m_{0}=m$, and let $m_{00}, m_{01}, m_{02}, \cdots$ be the other components of $\mathrm{Bd} H_{0}$. Since by (4.6.1), (4.8.1), (4.7) and (5.14.5) we have $B \cap P_{0}\left(M_{0}\right)=m$, we let $U_{0}^{0}, U_{1}^{0}$ be the components of $S^{3}-X_{0}$ with Int $B \subset U_{0}^{0}$ and let $\Gamma_{0}=\{0\}$. From the conclusion of Lemma 5.15 it follows that $\Gamma_{0}$, 4-tuple $\left(m_{0}, H_{0}, M_{0}, S_{0}\right)$, and function $P_{0}$ satisfy (5.16.1), (5.16.3), (5.16.4), (5.16.5), (5.16.7), (5.16.8), (5.16.9), and (5.16.10). Condition (5.16.2) is satisfied with the exception of the requirement $E=\bigcup_{\alpha \in \Gamma_{0}} H_{\alpha}$ and condition (5.16.6) is satisfied for $P \mid D_{0}^{*}$ where $D_{0}^{*}=X_{0}-(T-D)$ and $\bar{D}_{0}^{*}=D_{0}^{*} \bmod S_{0} \cup E$. The conclusion of Lemma 5.15 shows that the following properties are also satisfied.

$\left(a_{0}\right) \quad P_{0}\left(M_{0}\right) \cap\left(X_{0}-M_{0}\right)=\varnothing$,

$\left(b_{0}\right)$ if $\sigma \in K_{3}(0)$ then $P_{0} \mid \sigma$ is a separation isomorphism from $\bar{\sigma}^{\prime}$ to $\left\{P_{0}(\tau) \mid \tau \in \bar{\sigma}^{\prime}\right\}$, and

$\left(c_{0}\right)$ if $\sigma_{0}, \sigma_{1} \in K_{3}(0)$ and $\sigma_{0} \neq \sigma_{1}$ but $P_{0}\left(\sigma_{0}\right) \subset P_{0}\left(\sigma_{1}\right)$ then there exists $\sigma_{2} \in K_{3}^{\prime}(0)$ such that $\sigma_{2} \subset \sigma_{1}$ and $P_{0}\left(\sigma_{0}\right) \subset P_{0}\left(\sigma_{2}\right)$.

We proceed inductively up the lexicograpical ordering of the $m_{\alpha}$ 's. Suppose for each element $\alpha$ of a tree labeling system $\Gamma_{k}$, a 4-tuple 
$\left(m_{\alpha}, H_{\alpha}, M_{\alpha}, S_{\alpha}\right)$, and function $P_{k}$ from $S^{3}$ to $S^{3}$ exist that satisfy (5.16.1), (5.16.2) as modified above, (5.16.3), $\cdots,(5.16 .5),(5.16 .6)$ as modified above, (5.16.7), $\cdots,(5.16 .10)$, and,

$\left(a_{k}\right) \quad P_{k}\left(\cup\left\{M_{\alpha} \mid \alpha \in \Gamma_{k}\right) \cap\left(X_{k}-\cup\left\{M_{\alpha} \mid \alpha \in \Gamma_{k}\right\}\right)=\varnothing\right.$

$\left(b_{k}\right)$ if $\sigma \in K_{3}(k)$ then $P_{k} \mid \sigma$ is a separation isomorphism from $\bar{\sigma}^{\prime}$ to $\left\{P_{k}(\tau) \mid \tau \in \bar{\sigma}^{\prime}\right\}$, and

$\left(c_{k}\right)$ if $\sigma_{0}, \sigma_{1} \in K_{3}(k)$ and $\sigma_{0} \neq \sigma_{1}$ but $P_{k}\left(\sigma_{0}\right) \subset P_{k}\left(\sigma_{1}\right)$ then there exists $\sigma_{2} \in K_{3}^{\prime}(k)$ such that $\sigma_{2} \subset \sigma_{1}$ and $P_{k}\left(\sigma_{0}\right) \subset P_{k}\left(\sigma_{2}\right)$, where $K(k)=K_{1}(k) \cup K_{2}(k) \cup K_{3}(k)=\cup\left\{K(\alpha) \mid \alpha \in \Gamma_{k}\right\}, K^{\prime}(k)=K_{1}^{\prime}(k) \cup$ $K_{2}^{\prime}(k) \cup K_{3}^{\prime}(k)$ is the subdivision of $K(k)$ given by $K(k) \bmod \left(X_{k}-\cup\right.$ $\left.\left\{\operatorname{Bd} M_{\alpha}-\operatorname{Int} H_{\alpha} \mid \alpha \in \Gamma_{k}\right\}\right), D_{k}^{*}=X_{k}-(T-D)$, and $\bar{D}_{k}^{*}=D_{k}^{*} \bmod \left(\cup\left\{S_{\alpha} \mid \alpha \epsilon\right.\right.$ $\left.\left.\Gamma_{k}\right\}\right) \cup E$. Let $U_{0}^{k}, U_{1}^{k}$ be the components of $S^{3}-X_{k}$ where Int $B \subset U_{0}^{k}$.

Let $\Gamma_{k+1}=\Gamma^{k} \cup\{\beta\}$ where $\beta$ is the next element in the lexicographical ordering of the $m_{\alpha}$ 's. That is, if $\alpha=i_{1} i_{2} \cdots i_{n}$ is the last element of $\Gamma_{k}$ then $\beta=i_{1} i_{2} \cdots i_{n-1}\left(i_{n}+1\right)$ or $\beta=i_{1} i_{2} \cdots i_{n} 0$ depending on which of these is the lowest subscript among the symbols labeling the boundary components of the desk-with-holes $\cup\left\{H_{\alpha} \mid \alpha \in \Gamma_{k}\right\}$. Let $a_{k+1}=\left(X_{k}, U_{|\beta|}^{k}, q, m_{\beta}\right)$ and apply Lemma 5.15 to obtain 5-tuple $\left(H_{\beta}, M_{\beta}\right.$, $\left.\hat{S}, \hat{X}_{k+1}, \hat{P}\right)$ that satisfies the conclusion of Lemma 5.15 with $\hat{K}=\hat{K}_{1} \cup$ $\hat{K}_{2} \cup \hat{K}_{3}$ the separation complex supported by $\hat{S}$ in $M_{\beta}$. Properties (5.16.1), (5.16.2) as modified above, and (5.16.3) and (5.16.3) are easily established and we leave this to the reader. Label the components (if there are any) of $\left(\mathrm{Bd} H_{\beta}\right)-m_{\beta}$ with $m_{\beta 0}, m_{\beta 1}, \cdots$.

It follows by (5.14.2), (4.6.1), (4.8.1), (4.7), (5.16.2), and (5.16.4) that $\left(B \cup\left(\cup\left\{G_{\alpha} \mid \alpha \in \Gamma_{k}\right\}\right)\right) \cap \hat{P}\left(M_{\beta}\right)=m_{\beta}$ and $\hat{P} \mid\left(B \cup\left(\cup\left\{G_{\alpha} \mid \alpha \in \Gamma_{k}\right\}\right)\right)=1$ where $G_{\alpha}=\operatorname{Bd} M_{\alpha}-$ Int $H_{\alpha}$. Hence, $\hat{X}_{k+1}$ contains $(T-D) \cup\left(\cup\left\{G_{\alpha} \mid \alpha \in \Gamma_{k+1}\right\}\right)$.

For $\sigma \in \hat{K}_{3}, \hat{P}(\sigma)$ may intersect $\cup\left\{\operatorname{Int} M_{\alpha} \mid \alpha \in \Gamma_{k}\right\}$ : however, $\hat{P}(\sigma)$ may intersect at most one of the sets Int $M_{\alpha}, \alpha \in \Gamma_{k} \cdot$ Let $\alpha(\sigma)$ be the subscript such that $\hat{P}(\sigma) \cap \operatorname{Int} M_{\alpha(\sigma)} \neq \varnothing$. Proceeding as in Case B, paragraph 4, of the induction step in the proof of Lemma 5.15 we may apply Lemma 3.14 and Lemma 3.10 and assume without loss of generality that $S(\sigma)$ is a compact $P L$ 2-manifold in $\sigma$ such that if $Y$ is a component of $S(\sigma)$ then $Y \cap \mathrm{Bd} \sigma=Y \cap((\mathrm{Bd} \sigma)-\widehat{S})=\mathrm{Bd} Y \neq Q$ and $\hat{P}(Y)$ is a component of $S_{\alpha(\sigma)} \cap \hat{P}(\sigma)$. Let $S_{\beta}=\hat{S} \cup\left(\cup\left\{S(\sigma) \mid \sigma \in K_{3}\right\}\right)$. It is clear that $S_{\beta}$ partitions $M_{\beta}$ and let $K(\beta)=K_{1}(\beta) \cup K_{2}(\beta) \cup K_{3}(\beta)$ be the separation complex that $S_{\beta}$ supports in $M_{\beta}$, thus (5.16.4) is satisfied. It is clear that $\hat{P}$ satisfies (5.16.7) and (5.16.10) for $\sigma, \sigma_{0}$, $\sigma_{1} \in K_{3}(\beta)$ since $\hat{P}$ satisfied (4.6.2) and (4.6.5) for $\sigma, \sigma_{0}, \sigma_{1} \in K_{3}$ and the applications of Lemma 3.14 and Lemma 3.10 haven't altered these properties. Let $K(k+1)=K_{1}(k+1) \cup K_{2}(k+1) \cup K_{3}(k+1)=\cup\{K(\alpha) \mid \alpha \epsilon$ $\left.\Gamma_{k+1}\right\}=\hat{K}(k) \cup K(\beta)$, and Let $S_{k+1}=\cup\left\{S_{\alpha} \mid \alpha \in \Gamma_{k+1}\right\}=S_{k} \cup S_{\beta}$.

We now show that $P_{k} \cdot \hat{P} \mid M_{\beta}$ is winding function with respect to $\left(M_{\beta}, S_{\beta}, H_{\beta}, R\right)$. Property $(4.6 .1)$ is satisfied since by (5.14.2), and (5.16.5), respectively, $\hat{P}, P_{k}$ satisfy (4.6.1). Property (4.6.2) is estab- 
lished by observing that, by the way $K(\beta)$ was constructed, if $\sigma \in$ $K_{3}(\beta)$ then $\left(\cup\left\{M_{\alpha} \mid \alpha \in \Gamma_{k}\right\}\right) \cap \operatorname{Int} \hat{P}(\sigma)=\varnothing$ or $\hat{P}(\sigma)=|L|$ where $L$ is a subcomplex of $\bar{\sigma}_{0}^{\prime}$ for some $\sigma_{0} \in K(k)$ ( $\bar{\sigma}_{0}^{\prime}$ is the induced subdivision of $\bar{\sigma}_{0}$ by $\left.K^{\prime}(k)\right)$. In the former case, (4.6.2) follows by (5.16.5) and in the latter case by $\left(b_{k}\right)$. Properties (4.6.3) and (4.6.4) are straight forward consequences of the corresponding properties for $\hat{P}, \quad(5.16 .8)$ and (5.16.9) for $P_{k}$, and the way $S_{\beta}$ was constructed. We now establish property (4.6.5). Suppose $\sigma_{0}, \sigma_{1} \in K_{3}(\beta), \sigma_{0} \neq \sigma_{1}$, and $P_{k} \hat{P}\left(\sigma_{0}-S_{\beta}\right) \cap$ $P_{k} \hat{P}\left(\sigma_{1}-S_{\beta}\right) \neq \varnothing$ then (a) $M_{\alpha} \cap \operatorname{Int} \hat{P}\left(\sigma_{i}\right)=\varnothing$ for $i=0,1$ and all $\alpha \epsilon$ $\Gamma_{k}$, or (b) $\hat{P}\left(\sigma_{i}\right) \subset M_{\alpha}, M_{\gamma} \cap \operatorname{Int} \hat{P}\left(\sigma_{1-i}\right)=\varnothing$ for some $\alpha$, and all $\gamma \in \Gamma_{k}$, or (c) $\hat{P}\left(\sigma_{i}\right) \subset M_{\alpha}, \hat{P}\left(\sigma_{1-i}\right) \subset M_{\gamma}$ for some $\alpha, \gamma \in \Gamma_{k}$. In Case (a), (4.6.5) follows from the corresponding properties for $\hat{P}$, the way $S_{\beta}$ was constructed, and (5.16.5). In Case (b), $P_{k} \hat{P}\left(\sigma_{i}-S_{\beta}\right) \subset \operatorname{Int} P_{k} \hat{P}\left(\sigma_{1-i}\right)$ since $P_{k} \hat{P}\left(\sigma_{1-i}-S_{\beta}\right) \subset X_{k}-\left(\cup\left\{M_{\alpha} \mid \alpha \in \Gamma_{k}\right\}\right), P_{k} \hat{P}\left(\sigma_{i}-S_{\beta}\right)$ is connected, and by $\left(a_{k}\right)$. In Case (c), either (c.1) there exists a $\sigma^{*} \in K_{3}(k)$ such that $\hat{P}\left(\sigma_{i}\right) \subset \sigma^{*}$ for $i=0,1$ or (c.2) there exist distinct $\sigma_{0}^{*}, \sigma_{1}^{*} \in K_{3}(k)$ such that $\hat{P}\left(\sigma_{i}\right) \subset \sigma_{1}^{*}$. In Case (c.1), (4.6.5) follows from the corresponding property for $\hat{P}$ and $\left(b_{k}\right)$. In Case (c.2), (4.6.5) follows from $\left(c_{k}\right)$, sice we must have $P_{k}\left(\sigma_{i}^{*}\right) \subset P_{k}\left(\sigma_{1-i}^{*}\right)$ for $i=0$, or 1 by (5.16.10).

Let $\hat{P}_{k+1}=P_{k} \hat{P}$. We showed in the previous paragraph that $\hat{P}_{k+1} \mid M_{\beta}$ is a winding function with respect to $\left(M_{\beta}, S_{\beta}, H_{\beta}, R\right)$. This, combined with (5.16.5), (5.16.7), (5.16.8) and (5.16.9) for $P_{k}$ establishes (5.16.5), (5.16.7), (5.16.8), and (5.16.9) for $\hat{P}_{k+1}$. Property, (5.16.6) is valid for $\hat{P}_{k+1}$ since it is valid for $P_{k}, \hat{P} \mid \cup\left\{G_{\alpha} \mid \alpha \in \Gamma_{k}\right\}=1$, and $\hat{P} \mid G_{\beta}$ is a separation isomorphism onto $J_{a_{k+1}}$ of Lemma 5.15.

Property (5.16.10) follows by the corresponding property for $P_{k}$ or $\hat{P}$ if either $\sigma_{0}, \sigma_{1} \in K_{3}(k)$ or $\sigma_{0}, \sigma_{1} \in K_{3}(\beta)$. The other cases are (a) $\sigma_{0} \in$ $K_{3}(k), \sigma_{1} \in K_{3}(\beta)$, and $\hat{P}\left(\sigma_{1}\right) \cap$ Int $M_{\alpha}=\varnothing$ for all $\alpha \in \Gamma_{k}$ or (b) $\sigma_{0} \in K_{3}(k)$, $\sigma_{1} \in K_{3}(\beta)$, and $\hat{P}\left(\sigma_{1}\right) \subset M_{\alpha}$ for some $\alpha \in \Gamma_{k}$. In Case (a), $\hat{P}_{k+1}\left(\sigma_{0}-S_{k+1}\right) \subset$ Int $\hat{P}_{k+1}\left(\sigma_{1}\right)$, and $\hat{P}_{k+1}\left(\sigma_{0} \cap\left(S_{k+1} \cup E\right)\right) \subset \operatorname{Int} \hat{P}_{k+1}\left(\sigma_{1} \cap\left(S_{k+1} \cup E\right)\right)$ since $\hat{P}_{k+1}\left(\sigma_{1}-S_{k+1}\right) \subset X_{k}-\left(\cup\left\{M_{\alpha} \mid \alpha \in \Gamma_{k}\right\}\right), \hat{P}_{k+1}\left(\sigma_{0}-S_{k+1}\right)$ is connected and by $\left(a_{k}\right)$. In Case (b) either (b.1) $\hat{P}\left(\sigma_{1}\right) \subset \sigma_{0}$ or (b.2) there exists a $\sigma^{*} \in$ $K_{3}(k)$ such that $\hat{P}\left(\sigma_{1}\right) \subset \sigma^{*}$. In Case (b.1) (5.16.10) follows by $b_{k}$ since $\hat{P}\left(\sigma_{1}\right)$ must be the underlying set of a subcomplex of $K^{\prime}(k)$ in $\sigma^{*}$. In Case (b.2), (5.16.10) follows from $c_{k}$, since we must have either $P_{k}\left(\sigma^{*}\right) \subset$ $P_{k}\left(\sigma_{0}\right)$, or $P_{k}\left(\sigma_{0}\right) \subset P_{k}\left(\sigma^{*}\right)$ by $(5.16 .10)$ for $P_{k}$.

The properties remaining to be varified are $\left(a_{k+1}\right),\left(b_{k+1}\right)$, and $\left(c_{k+1}\right)$. We establish these by using the lifting property for winding functions in Lemma 4.9. For $\sigma \in K_{3}(k+1)$ let $N(\sigma)=\left\{\tau \in K_{3}(k+1) \mid \hat{P}_{k+1}(\tau) \subset \hat{P}_{k+1}(\sigma)\right.$ and $\tau \neq \sigma\}$ and let $W(\sigma)=\hat{P}_{k+1}\left(\hat{X}_{k+1}-\left(\cup\left\{G_{\alpha} \mid \alpha \in \Gamma_{k+1}\right\}\right)\right) \cap\left(\hat{P}_{k+1}(\sigma)-\cup\right.$ $\left.\left\{\hat{P}_{k+1}(\tau) \mid \tau \in N(\sigma)\right\}\right)$. It is clear that $\left(\hat{P}_{k+1}\left(\cup\left\{M_{\alpha} \mid \alpha \in \Gamma_{k+1}\right\}\right)-\hat{P}_{k+1}\left(\cup\left\{G_{\alpha} \mid \alpha \in\right.\right.\right.$ $\left.\left.\left.\Gamma_{k+1}\right\}\right)\right) \cap \hat{P}_{k+1}\left(\hat{X}_{k+1}\right)=\cup\left\{W(\sigma) \mid \sigma \in K_{3}(k+1)\right\}$ and if $\sigma_{0} \neq \sigma_{1}$ then Int $W\left(\sigma_{0}\right) \cap$ Int $W\left(\sigma_{1}\right)=\varnothing$. Also, by (5.16.8), (5.16.9), and (5.16.10), if $\sigma_{0} \cap \sigma_{1}$ is a component of $S_{k+1}$ then $W\left(\sigma_{0}\right) \cap W\left(\sigma_{1}\right)=\hat{P}_{k+1}\left(\hat{X}_{k+1}-\cup\left\{G_{\alpha} \mid \alpha \in\right.\right.$ 
$\left.\left.\Gamma_{k+1}\right\}\right) \cap\left(\hat{P}_{k+1}\left(\sigma_{0} \cap \sigma_{1}\right)-\cup\left\{\hat{P}_{k+1}(\tau) \mid \tau \in N\left(\sigma_{i}\right)\right\}\right)$ for $i=0$ or 1 . It follows that for each $\alpha \in \Gamma_{k+1}, W_{\alpha}=\cup\left\{W(\sigma) \mid \sigma \in K_{3}(\alpha)\right\}$ is a compact 2-manifold that satisfies the hypothesis of Lemma 4.9 along with winding function $\hat{P}_{k+1} \mid M_{\alpha}$ with respect to $\left(M_{\alpha}, S_{\alpha}, H_{\alpha}, R\right)$. Apply Lemma 4.9 for each $\alpha \in \Gamma_{k+1}$ and let $\bar{W}_{\alpha}$ be the resulting compact 2-manifold, $K^{\prime}(\alpha)$ the resulting subdivision of $K(\alpha)$ satisfying 4.9.3 and $P_{k+1} \mid M_{\alpha}$ the resulting function that satisfies $(4.9 .1), \cdots,(4.9 .6)$.

Let $X_{k+1}=\left(\cup\left\{\bar{W}_{\alpha} \mid \alpha \in \Gamma_{k+1}\right\}\right) \cup\left(\cup\left\{G_{\alpha} \mid \alpha \in \Gamma_{k+1}\right\}\right) \cup\left(\hat{X}_{k+1}-\hat{P}_{k+1}(\cup\right.$ $\left.\left.\left\{M_{\alpha} \mid \alpha \in \Gamma_{k+1}\right\}\right)\right)$, let $\left.P_{k+1} \mid \cup\left\{M_{\alpha} \mid \alpha \in \Gamma_{k+1}\right\}=\bigcup\left\{\left(P_{k+1} \mid M_{\alpha}\right) \mid \alpha \in \Gamma_{k+1}\right)\right\}$ and let $K^{\prime}(k+1)=K_{1}^{\prime}(k+1) \cup K_{2}^{\prime}(k+1) \cup K_{3}^{\prime}(k+1)=\bigcup\left\{K^{\prime}(\alpha) \mid \alpha \in \Gamma_{k+1}\right\}$. By (4.9.1), $P_{k+1}$ satisfies (5.16.5), $\cdots,(5.16 .10)$, thus it is only necessary to varify $\left(a_{k+1}\right),\left(b_{k+1}\right)$, and $\left(c_{k+1}\right)$. Property $\left(a_{k+1}\right)$ follows since $X_{k+1}-$ $\bigcup\left\{M_{\alpha} \mid \alpha \in \Gamma_{k+1}\right\}=\hat{X}_{k+1}-\hat{P}_{k+1}\left(\bigcup\left\{M_{\alpha} \mid \alpha \in \Gamma_{k+1}\right\}\right)$. Property $\left(b_{k+1}\right)$ follows by (4.9.4). Property $\left(c_{k}\right)$ is established by observing that if $P_{k+1}\left(\sigma_{0}\right) \subset$ $P_{k+1}(\sigma)$ then $P_{k+1}\left(\sigma_{0}\right)$ is in a component $Z$ of $P_{k+1}\left(\sigma_{1}\right)-W\left(\sigma_{1}\right)$ since $P_{k+1}\left(\sigma_{0}\right)$ is connected and $W\left(\sigma_{1}\right) \cap P_{k+1}\left(\sigma_{0}\right)=\varnothing$ by definition of $W\left(\sigma_{1}\right)$. The lift of $\mathrm{Cl} Z$ is the required $\sigma_{2}$, that is, $\sigma_{2}=\left(P_{k+1} \mid \sigma_{1}\right)^{-1}(\mathrm{Cl} Z)$.

The induction step is now complete. The above process terminates when $\cup\left\{H_{\alpha} \mid \alpha \in \Gamma_{k}\right\}$ is the disk $E$, thus completing property (5.16.2). The reason for not including properties $\left(a_{k}\right),\left(b_{k}\right),\left(c_{k}\right)$ in the conclusion to Theorem 5.16 is that they are "empty" in the sense that $(\bar{D} \cup$ $(T-D)) \cap\left(\cup\left\{\operatorname{Int} M_{\alpha} \mid \alpha \in \Gamma\right\}\right)=\varnothing$.

6. Disjoint singular disks. We now prove the following corollary to $\S \S 3,4$, and 5 .

Corollary 6.1. Suppose $T$ is a 2-sphere is $S^{3}, D$ is a $P L$ subdisk of $T$, and $E$ is a $P L$ disk in $S^{3}$ in general position with respect to $T$ such that $E \cap T \subset D$ and $\mathrm{Bd} E=\mathrm{Bd} D$. Let $U_{0}, U_{1}$ be the components of $S^{3}-T$ and suppose that $E_{i}(i=0,1)$ is a $P L$ disk in general position with respect to $D$ such that $\mathrm{Bd} E_{i} \subset U_{i}, E_{i}$ fails to intersect the 2-sphere $E \cup(T-D)$, and $T-D, \mathrm{Bd} E_{0}, \mathrm{Bd} E_{1}$ are in the same component of $S^{3}-(D \cup E)$. Let $F_{i}(i=0,1)$ be the component of $E_{i} \cap U_{i}$ that contains $\mathrm{Bd} E_{i}$. Then for $i=0,1$ there exist collections $\mathscr{D}_{i}, \mathscr{C}_{i}$ of components of $D-E_{i}$ and of $E_{i} \cap U_{i}$ respectively such that

(6.1.1) if $A \in \mathscr{D}_{0}, B \in \mathscr{D}_{1}$ then $\mathrm{Cl} A \cap \mathrm{Cl} B=\varnothing$,

(6.1.2) if $A \in \mathscr{E}_{i}$ and $B \in \mathscr{D}_{1-i}$ then $E$ separates $A$ from $B$ in $\mathrm{Cl} U_{i}$, and

(6.1.3) the set $D_{i}=\cup\left\{\mathrm{Cl} A \mid A \in \mathscr{D}_{i} \cup \mathscr{E}_{i}\right\}$ is a singular disk with $\mathrm{Bd} D_{i}=\mathrm{Bd} E_{i}$ obtained by pasting in the sense that there exists a map $f_{i}: E_{i} \rightarrow D_{i}$ such that $f_{i} \mid F_{i}=1$ and if $K$ is any component of $f_{i}^{-1}(\mathrm{Cl} A)$ for $A \in \mathscr{D}_{i} \cup \mathscr{E}_{i}$ then $f_{i} \mid K$ is a PL homeomorphism onto $\mathrm{Cl} A$. 
Proof. It follows from the polyhedral approximation theorem of Bing [1] that we may assume that the 2-sphere $T$ is piecewise linear. In order to use Theorem 5.16, we assume that the component of $S^{3}-T$ have been labeled so that $\mathrm{Cl}\left(U_{1}\right)$ contains a collar of $\mathrm{Bd} E$ in $E$ and $B$ is a collar of $\mathrm{Cl}(T-D)$ in $\mathrm{Cl} U_{0}$ such that $B \cap E=\mathrm{Bd} E$, and for $i=0$, or $1, B \cap E_{i}=\varnothing$. Let $R$ be the 2 -sphere defined by $R=E \cup((\operatorname{Bd} B)-(T-D))$ and let the components of $S^{3}-R$ be $V_{0}$, $V_{1}$ where Int $B \subset V_{0}$. It follows that $B-m, \mathrm{Bd} E_{0}, \mathrm{Bd} E_{1}$ are in the same component of $S^{3}-(D \cup E)$. We use the notation of Theorem 5.16, in particular let $\Gamma, m_{\alpha}, H_{\alpha}, M_{\alpha}, S_{\alpha}, K(\alpha), K$ and $P$ be as in the conclusion. The map $f_{i}$ is found by cutting $E_{i}$ off on $\cup\left\{P\left(\left(\mathrm{Bd} M_{\alpha}\right)-\right.\right.$ $\left.\left.H_{\alpha}\right)|\alpha \in \Gamma,| \alpha \mid=1-i\right\}$, but first we observe some useful facts.

It follows from (5.16.2), (5.16.3) that

(6.1.4) $\quad M_{\alpha}$ contains a corner in $\mathrm{Cl}\left(U_{|\alpha|} \cap V_{|\alpha|}\right)$. Also, by (5.16.5), (4.8.2) we have that

(6.1.5) $P\left(M_{\alpha}\right)$ contains a collar of $P\left(\mathrm{Bd} M_{\alpha}\right.$ - Int $\left.H_{\alpha}\right)$ in $\mathrm{Cl} U_{|\alpha|}$.

By (5.16.5), (5.16.7), $\cdots,(5.16 .10)$, respectively, we have, respectively, (4.6.1), (4.6.2), $\cdots,(4.6 .5)$. Thus $P \mid M_{\alpha}$ is a winding function with respect to $\left(M_{\alpha}, S_{\alpha}, H_{\alpha}, R\right)$; consequently, by (4.8.1) we have

(6.1.6) $\left((B-m) \cup \operatorname{Bd} E_{0} \cup \operatorname{Bd} E_{1}\right) \cap P\left(M_{\alpha}\right)=\varnothing$ for all $\alpha \in \Gamma$.

We wish to establish that

(6.1.7) $\quad E_{i} \cap U_{1-i} \subset E_{i} \cap P\left(\cup\left\{M_{\alpha} \mid \alpha \in \Gamma\right\}\right) \subset E_{i} \cap P\left(\cup\left\{M_{\alpha}|\alpha \in \Gamma| a \mid,=\right.\right.$ $1-i\})$ for $i=0$ or 1 .

Let $A=\left\{Y \mid Y\right.$ is a component of $E_{i} \cap U_{1-i}$ such that there does not exist a $\sigma \in K_{3}$ such that $\left.Y \subset P(\sigma)\right\}$ and let $B=\{Z \mid Z$ is a component of $E_{i} \cap \operatorname{Int} P(\sigma)$ where $\sigma \in \cup\left\{K_{3}(\alpha)|\alpha \in \Gamma,| \alpha \mid=1\right\}$ and there does not exist a $\tau \in \cup\left\{K_{3}(\alpha)|\alpha \in \Gamma,| \alpha \mid=1-i\right\}$ such that $\left.P(\sigma) \subset P(\tau)\right\}$. Suppose $A \cup B \neq \varnothing$, then there exists an outermost element of $A \cup B$ in $E_{i}$. That is, there exists an element $W \in A \cup B$ such that $W^{\prime}$ fails to separate $W$ from $\operatorname{Bd} E_{i}$ in $E_{i}$ for all $W^{\prime} \in A \cup B$. Let $w$ be the outermost component of $\mathrm{Bd} W$ and let $X$ be the component of $E_{i}-T$ such that $w \subset \operatorname{Bd} X$ but $X \cap W=\varnothing$. If $W \in A$ then, since $w \subset P(\operatorname{Bd} \sigma-$ $(S \cup E)$ ) for some $\sigma \in K_{3}$ and because of (6.1.5), it follows that there exists a $Z \in B$ such that $X \subset Z$ contradicting that $W$ is an outermost member of $A \cup B$ in $E_{i}$. If $W \in B$, then, by (6.1.5) since $W$ is also an outermost member of $B$, it follows that $X \in A$ contradicting that $W$ is an outermost member of $A \cup B$ in $E_{i}$. Hence we must have $A \cup B=\varnothing$ and (6.1.7) is established.

We wish now to establish that

(6.1.8) $E$ separates $E_{i}-P\left(\cup\left\{M_{\alpha}|\alpha \in \Gamma,| \alpha \mid=1-i\right\}\right)$ from $P(\cup$ $\left.\left\{\left(\operatorname{Bd} M_{\alpha}\right)-H_{\alpha}|\alpha \in \Gamma,| \alpha \mid=i\right\}\right)$ in $\mathrm{Cl} U_{i}$.

Suppose (6.1.8) is false, then by (6.1.7) there exists an arc $a b$ from $a$ to $b$ such that $a \in E_{i}-P\left(\cup\left\{M_{\alpha} \mid \alpha \in \Gamma\right\}\right), b \in P\left(\left(\operatorname{Bd} M_{\alpha}\right)-H_{\alpha}\right)$ for some $\alpha \in \Gamma$ with $|\alpha|=i$, and Int $a b \subset U_{i}-E$. However, by (6.1.5) 
there exists a point $c$ in $a b$ near $b$ such that $c \in\left(P\left(M_{\alpha}\right)-P\left(\operatorname{Bd} M_{\alpha}\right)\right) \cap$ $U_{i}$. But this contradicts (4.8.1), thus (6.1.8) is established.

The collections $\mathscr{E}_{i}, \mathscr{D}_{i}(i=0,1)$ of the conclusion will be chosen such that $\cup\left\{A \mid A \in \mathscr{E}_{i}\right\} \subset E_{i}-P\left(\cup\left\{M_{\alpha}|\alpha \in \Gamma,| \alpha \mid=1-i\right\}\right)$ and $\cup\{\mathrm{Cl} A \mid A \in$ $\left.\left.\mathscr{D}_{i}\right\} \subset P\left(\cup\left\{\operatorname{Bd} M_{\alpha}\right)-H|\alpha \in \Gamma,| \alpha \mid=1-i\right\}\right)$. Properties (6.1.2) and (6.1.1) will then follow by (6.1.8) and the fact that $P\left(\cup\left\{\left(\mathrm{Bd} M_{\alpha}\right)-H_{\alpha} \mid \alpha \in\right.\right.$ $\Gamma,|\alpha|=i\}) \cap P\left(\cup\left\{\left(\operatorname{Bd} M_{\alpha}\right)-H_{\alpha}|\alpha \in \Gamma,| \alpha \mid=1-i\right\}\right)=\varnothing$.

If $m$ is a component of $D \cap E_{i}$ then we let $E_{i}(m)$ be the disk $m$ bounds is $E_{i}$. Let $\mathscr{E}_{i}^{\prime}=\left\{X \mid X\right.$ is a component of $E_{i}-P\left(\cup\left\{M_{\alpha}|\alpha \in \Gamma,| \alpha \mid=\right.\right.$ $1-i\})$. Each member of $\mathscr{E}_{i}^{\prime}$ is an open disk-with-holes whose boundary consists of one "outer" simple closed curve and perhaps several "inner" simple closed curves. We accordingly let $\mathcal{O}_{i}=\{m \mid m$ is a component of $\mathrm{Bd} X$ for some $X \in \mathscr{E}_{i}^{\prime}$, and $\left.X \subset E_{i}(m)\right\}$ and $\mathscr{I}_{i}=\{m \mid m$ is a component of $\mathrm{Bd} X$ for some $X \in \mathscr{E}_{i}^{\prime}$, and $\left.X \cap E_{i}(m)=\varnothing\right\}$. For each $m \in$ $\mathscr{S}_{i}$ we find a disk-with-holes $D(m) \subset P\left(\cup\left\{\left(\operatorname{Bd} M_{\alpha}\right)-H_{\alpha}|\alpha \in \Gamma,| \alpha\right\}=\right.$ $1-i\})$ such that $m \subset \operatorname{Bd} D(m)$ and if $u$ is a component of $(\operatorname{Bd} D(m))$ $m$ then $u \in \mathcal{O}_{i}$ and $u \subset E_{i}(m)$. The $D(m)$ 's bridge the gaps between elements of $\mathscr{C}_{i}^{\prime}$ and enable us to define the map $f_{i}$ of the conclusion (see Figure 5).

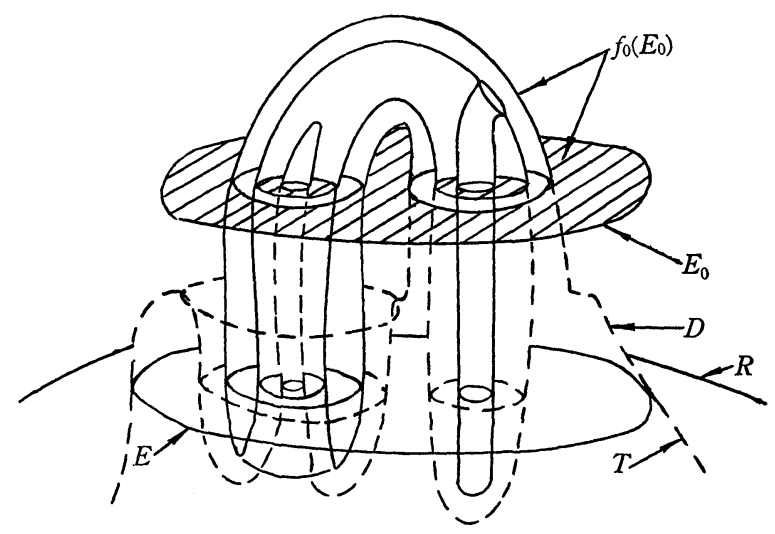

FIGURE 5

Let $m \in \mathscr{F}_{i}$; then $m \subset P(\mathrm{Bd} \sigma-(S \cup E))$ for a unique $\sigma \in K_{3}(\alpha)$ with $|\alpha|=1-i$. Let $Y$ be the component of $P(\sigma) \cap E_{i}$ that contains $m$. Clearly $Y \subset E_{i}(m)$, and since there does not exist a $\tau \in K_{3}$ such that $P(\sigma) \subset$ Int $P(\tau)$ it follows by (6.1.5) that each component of $(\mathrm{Bd} Y)-m$ is in $\mathcal{O}_{i}$. Since each component $t$ of $\mathrm{Bd} Y$ separates $T$, we have that $t$ separates the 2-face $\tau$ of $\{P(\tau) \mid \tau \in \bar{\sigma}\}$ that contains $t$. By Lemma 3.14, $Y$ separates $P(\sigma)$, and by Lemma 3.10 we may assume without loss of generality that there exists a compact, connected $P L$ 2-manifold $\sigma_{0} \subset \sigma$ such that $\sigma_{0} \cap \mathrm{Bd} \sigma=\sigma_{0} \cap((\mathrm{Bd} \sigma)-(S \cup E))=\mathrm{Bd} \sigma_{0}$ and $P \mid \sigma_{0}$ is a separation isomorphism from $\bar{\sigma}_{0}$ to $\{t \mid t$ is a component of $\mathrm{Bd} Y\} \cup\{Y\}$. Let $m^{\prime}$ be the component of $\operatorname{Bd} \sigma$ such that $P\left(m^{\prime}\right)=m$. The 2-mani- 
fold $\sigma_{0}$ separates $M(\alpha)$ so let $D^{\prime}(m)$ be the component of $(\operatorname{Bd} M(\alpha))-\sigma_{0}$ that contains $m^{\prime}$ in its boundary but does not contain $H(\alpha)$. It follows that $\mathrm{Bd} D^{\prime}(m) \subset \mathrm{Bd} \sigma_{0}$. Let $D(m)=P\left(D^{\prime}(m)\right)$.

For $m_{\alpha} \in \mathcal{O}_{i}$, let $X\left(m_{\alpha}\right)$ be the element of $\mathscr{E}_{1}^{\prime}$ that contains $m_{\alpha}$ in its boundary and let $m_{\alpha 0}, m_{\alpha 1}, \ldots$ be the remaining components of $\operatorname{Bd} X\left(m_{\alpha}\right)$. Also, let $m_{\beta 0}, m_{\beta 1}, \cdots$ be the components of $\left(\operatorname{Bd} D\left(m_{\beta}\right)\right)-m_{\beta}$. Let $m_{0}^{i}=\mathrm{Bd} E_{i}$, then for $i=0,1, f_{i}, \mathscr{E}_{i}, \mathscr{D}_{i}$ of conclusion are given respectively by

$$
\begin{aligned}
f_{i}\left(E_{i}\right) & =X\left(m_{0}^{i}\right) \cup\left(\bigcup_{i_{1}} D\left(m_{0 i_{1}}\right)\right) \cup\left(\bigcup_{i_{1}, i_{2}} X\left(m_{0 i_{1} i_{2}}\right)\right) \cup\left(\bigcup_{i_{1}, i_{2}, i_{3}} D\left(m_{0 i_{1}, i_{2}, i_{3}}\right)\right) \cup \cdots \\
\mathscr{E}_{i} & =\left\{X\left(m_{0}^{i}\right)\right\} \cup\left\{X\left(m_{0 i_{1} i_{2}}\right)\right\} \cup\left\{X\left(m_{0 i_{1} i_{2} i_{3} i_{4}}\right)\right\} \cup \cdots \\
\mathscr{D}_{i} & =\left\{D\left(m_{0 i_{1}}\right)\right\} \cup\left\{D\left(m_{0 i_{1} i_{2} i_{3}}\right)\right\} \cup\left\{D\left(m_{0 i_{1} i_{2} i_{3} i_{4} i_{5}}\right)\right\} \cup \cdots
\end{aligned}
$$

That there exists no circularity in $f_{i}\left(E_{i}\right)$ follows from the fact that $\operatorname{Bd} D\left(m_{\alpha}\right) \subset E_{i}\left(m_{\alpha}\right)$ or, in other words, we proceed always from the "outside" to the "inside" along $E_{i}$.

\section{REFERENCES}

1. R. H. Bing, Approximating surfaces with polyhedral ones, Ann. of Math., (2) 65 (1957), 456-483.

2. ㄴ. Approximating surfaces from the side, Ann. of Math., (2) 77 (1963), 145192.

3. - Improving the side approximation theorem, Trans. Amer. Math. Soc., 116 (1965), 511-525.

4. - Each disk in $E^{3}$ contains a tame arc, Amer. J. Math., 84 (1962), 583-590.

5. —_, Pushing a 2-sphere into its complement, Mich. Math. J., 11 (1964), 33-45.

6. - Improving the intersection of lines and surfaces, Michigan Math. J., 14 (1967), 155-159.

7. W. T. Eaton, The sum of solid spheres, Michigan Math. J., 19 (1972), 193-207.

8. - Applications of a mis-match theorem to decomposition spaces, to appear.

9. F. M. Lister, Simplifying intersections of disks in Bing's side approximation theorem, Pacific J. Math., 22 (1967), 281-295.

10. C. D. Papakyriakopoulos, On Dehn's lemma and the asphericity of knots, Ann. of Math., (2) 66 (1957), 1-26.

Received September 28, 1971 and in revised form June 28, 1972. This research was supported by the Alfred P. Sloan Foundation.

The Institute For Advanced Study

AND

The University of Texas 



\section{PACIFIC JOURNAL OF MATHEMATICS}

\section{EDITORS}

\section{H. SAMELSON}

Stanford University

Stanford, California 94305

C. R. Новву

University of Washington Seattle, Washington 98105

\section{J. DuGundJI}

Department of Mathematics University of Southern California Los Angeles, California 90007

RICHARD ARENS

University of California Los Angeles, California 90024

\section{ASSOCIATE EDITORS}
E. F. BECKENBACH
B. H. NeumanN
F. WOLF
K. YoSHIDA

\section{SUPPORTING INSTITUTIONS}

\author{
UNIVERSITY OF BRITISH COLUMBIA \\ CALIFORNIA INSTITUTE OF TECHNOLOGY \\ UNIVERSITY OF CALIFORNIA \\ MONTANA STATE UNIVERSITY \\ UNIVERSITY OF NEVADA \\ NEW MEXICO STATE UNIVERSITY \\ OREGON STATE UNIVERSITY \\ UNIVERSITY OF OREGON \\ OSAKA UNIVERSITY
}

\author{
UNIVERSITY OF SOUTHERN CALIFORNIA \\ STANFORD UNIVERSITY \\ UNIVERSITY OF TOKYO \\ UNIVERSITY OF UTAH \\ WASHINGTON STATE UNIVERSITY \\ UNIVERSITY OF WASHINGTON \\ $*{ }^{*}$
AMERICAN MATHEMATICAL SOCIETY
NAVAL WEAPONS CENTER
}

The Supporting Institutions listed above contribute to the cost of publication of this Journal, but they are not owners or publishers and have no responsibility for its content or policies.

Mathematical papers intended for publication in the Pacific Journal of Mathematics should be in typed form or offset-reproduced, (not dittoed), double spaced with large margins. Underline Greek letters in red, German in green, and script in blue. The first paragraph or two must be capable of being used separately as a synopsis of the entire paper. The editorial "we" must not be used in the synopsis, and items of the bibliography should not be cited there unless absolutely necessary, in which case they must be identified by author and Journal, rather than by item number. Manuscripts, in duplicate if possible, may be sent to any one of the four editors. Please classify according to the scheme of Math. Rev. Index to Vol, 39. All other communications to the editors should be addressed to the managing editor, Richard Arens, University of California, Los Angeles, California, 90024.

50 reprints are provided free for each article; additional copies may be obtained at cost in multiples of 50 .

The Pacific Journal of Mathematics is issued monthly as of January 1966. Regular subscription rate: $\$ 48.00$ a year (6 Vols., 12 issues). Special rate: $\$ 24.00$ a year to individual members of supporting institutions.

Subscriptions, orders for back numbers, and changes of address should be sent to Pacific Journal of Mathematics, 103 Highland Boulevard, Berkeley, California, 94708.

PUBLISHED BY PACIFIC JOURNAL OF MATHEMATICS, A NON-PROFIT CORPORATION

Printed at Kokusai Bunken Insatsusha (International Academic Printing Co., Ltd.), 270, 3-chome Totsuka-cho, Shinjuku-ku, Tokyo 160, Japan. 


\section{Pacific Journal of Mathematics}

\section{Vol. 44, No. $2 \quad$ June, 1973}

Tsuyoshi Andô, Closed range theorems for convex sets and linear liftings . . . . . . 393

Richard David Bourgin, Conically bounded sets in Banach spaces . . . . . . . . . 411

Robert Jay Buck, Hausdorff dimensions for compact sets in $R^{n} \ldots \ldots \ldots \ldots \ldots \ldots . \ldots 421$

Henry Cheng, A constructive Riemann mapping theorem ................ 435

David Fleming Dawson, Summability of subsequences and stretchings of

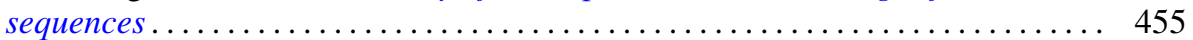

William Thomas Eaton, A two sided approximation theorem for 2-spheres ....... 461

Jay Paul Fillmore and John Herman Scheuneman, Fundamental groups of compact complete locally affine complex surfaces ....................... 487

Avner Friedman, Bounded entire solutions of elliptic equations . . . . . . . . . . . 497

Ronald Francis Gariepy, Multiplicity and the area of an $(n-1)$ continuous

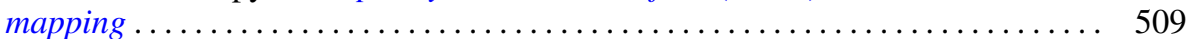

Andrew M. W. Glass, Archimedean extensions of directed interpolation groups . . . . 515

Morisuke Hasumi, Extreme points and unicity of extremum problems in $H^{1}$ on

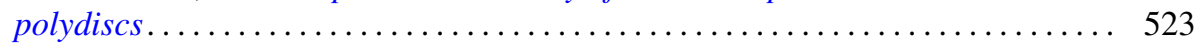

Trevor Ongley Hawkes, On the Fitting length of a soluble linear group . . . . . . 537

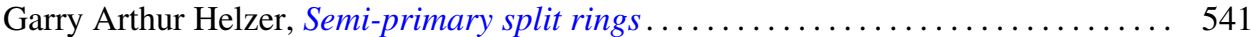

Melvin Hochster, Expanded radical ideals and semiregular ideals . . . . . . . . . 553

Keizō Kikuchi, Starlike and convex mappings in several complex variables . . . . . . 569

Charles Philip Lanski, On the relationship of a ring and the subring generated by its

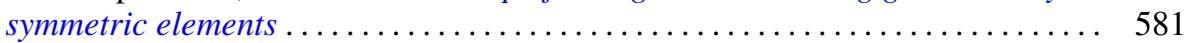

Jimmie Don Lawson, Intrinsic topologies in topological lattices and semilattices ........................................... 593

Roy Bruce Levow, Counterexamples to conjectures of Ryser and de Oliveira ...... 603

Arthur Larry Lieberman, Some representations of the automorphism group of an infinite continuous homogeneous measure algebra ..........

William George McArthur, $G_{\delta}$-diagonals and metrization theorems $\ldots .$.

James Murdoch McPherson, Wild arcs in three-space. II. An invariant of

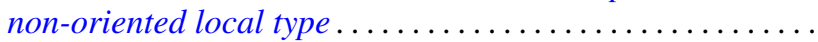

H. Millington and Maurice Sion, Inverse systems of group-valued measures ...

C. Edward Moore, Concrete semispaces and lexicographic separation of convex

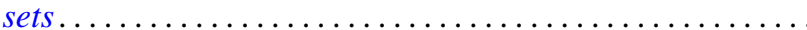

Jingyal Pak, Actions of torus $T^{n}$ on $(n+1)$-manifolds $M^{n+1}$.

Merrell Lee Patrick, Extensions of inequalities of the Laguerre and Turán type . . . . 675

Harold L. Peterson, Jr., Discontinuous characters and subgroups of finite index. . . . 683

S. P. Philipp, Abel summability of conjugate integrals . . . . . . . . . . . . . 693

R. B. Quintana and Charles R. B. Wright, On groups of exponent four satisfying an

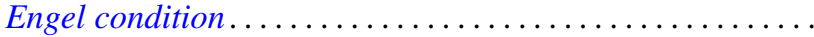

Marlon C. Rayburn, On Hausdorff compactifications. . . . . . . . . .

Martin G. Ribe, Necessary convexity conditions for the Hahn-Banach theorem in

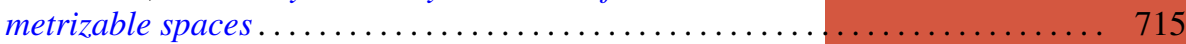

Ryōtarō Satō, On decomposition of transformations in infinite measure spaces .... 733

Peter Drummond Taylor, Subgradients of a convex function obtained from a

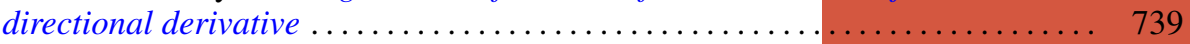

James William Thomas, A bifurcation theorem for $k$-set contractions . . . . . . . . 749 Clifford Edward Weil, A topological lemma and applications to real functions . . . . 757

Stephen Andrew Williams, A nonlinear elliptic boundary value problem . . ....... 767

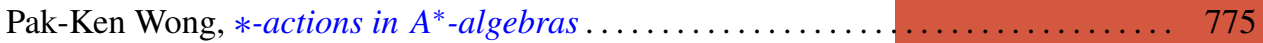

